Article

\title{
Genome-Wide Runs of Homozygosity, Effective Population Size, and Detection of Positive Selection Signatures in Six Chinese Goat Breeds
}

\author{
Rabiul Islam ${ }^{1,2}$, Yefang Li ${ }^{1}$, Xuexue Liu ${ }^{1}$, Haile Berihulay ${ }^{1}$, Adam Abied ${ }^{1} \mathbb{D}$, \\ Gebremedhin Gebreselassie ${ }^{1} \mathbb{D}$, Qing $\mathrm{Ma}^{3, *}$ and Yuehui Ma ${ }^{1, *}$ \\ 1 Institute of Animal Science (IAS), Chinese Academy of Agricultural Sciences (CAAS), No. 2 Yuanmingyuan \\ West Rd., Haidian, Beijing 100193, China; md.rabiul27@yahoo.com (R.I.); yefanglee1994@163.com (Y.L.); \\ Lxx_caas@163.com (X.L.); haile.berihulay@yahoo.com (H.B.); aa.abied89@gmail.com (A.A.); \\ gerageruggg@gmail.com (G.G.) \\ 2 Department of Livestock Services of Ministry of Fisheries and Livestock, Farmgate, Dhaka-1215, Bangladesh \\ 3 Institute of Animal Science, Ningxia Academy of Agriculture and Forestry Sciences, \\ Yinchuan 75002, Ningxia, China \\ * Correspondence: maqing1973@126.com (Q.M.); mayuehui@caas.cn (Y.M.)
}

Received: 21 October 2019; Accepted: 13 November 2019; Published: 17 November 2019

check for updates

\begin{abstract}
Detection of selection footprints provides insight into the evolution process and the underlying mechanisms controlling the phenotypic diversity of traits that have been exposed to selection. Selection focused on certain characters, mapping certain genomic regions often shows a loss of genetic diversity with an increased level of homozygosity. Therefore, the runs of homozygosity (ROHs), homozygosity by descent (HBD), and effective population size $\left(N_{e}\right)$ are effective tools for exploring the genetic diversity, understanding the demographic history, foretelling the signature of directional selection, and improving the breeding strategies to use and conserve genetic resources. We characterized the $\mathrm{ROH}, \mathrm{HBD}, N_{e}$, and signature of selection of six Chinese goat populations using single nucleotide polymorphism (SNP) 50K Illumina beadchips. Our results show an inverse relationship between the length and frequency of $\mathrm{ROH}$. A long $\mathrm{ROH}$ length, higher level of inbreeding, long HBD segment, and smaller $N_{e}$ in Guangfeng (GF) goats suggested intensive selection pressure and recent inbreeding in this breed. We identified six reproduction-related genes within the genomic regions with a high $\mathrm{ROH}$ frequency, of which two genes overlapped with a putative selection signature. The estimated pair-wise genetic differentiation $\left(F_{\mathrm{ST}}\right)$ among the populations is $9.60 \%$ and the inter- and intra-population molecular variations are $9.68 \%$ and $89.6 \%$, respectively, indicating low to moderate genetic differentiation. Our selection signatures analysis revealed 54 loci harboring 86 putative candidate genes, with a strong signature of selection. Further analysis showed that several candidate genes, including MARF1, SYCP2, TMEM200C, SF1, ADCY1, and BMP5, are involved in goat fecundity. We identified 11 candidate genes by using cross-population extended haplotype homozygosity (XP-EHH) estimates, of which MARF1 and SF1 are under strong positive selection, as they are differentiated in high and low reproduction groups according to the three approaches used. Gene ontology enrichment analysis revealed that different biological pathways could be involved in the variation of fecundity in female goats. This study provides a new insight into the ROHs patterns for maintenance of within breed diversity and suggests a role of positive selection for genetic variation influencing fecundity in Chinese goat.
\end{abstract}

Keywords: SNP; Chinese goat; genetic diversity; selection of signature; fecundity 


\section{Introduction}

Chinese goats have extensive genetic resources and an outstretched gene pool. Natural selection and artificial selection at different intensities over time, imposed by environmental changes and animal husbandry practices, have resulted in a considerable number of desirable traits such as extensive adaptability, outstanding prolificacy, and powerful disease and cold resistance [1]. The traceable unique genetic pattern remaining in the genomic regions of an individual under selection is termed their selection signature [2]. Selection focused on certain characters, mapping certain genomic regions, often shows reduced genetic diversity and stretches of homozygosity. Detection of this selection footmark in the genomic regions can provide information regarding the underlying genetic mechanisms of specific phenotypic traits to better guide animal breeding. The genetic diversity of animals is vital for promoting their current production potential in a diverse environment, changing breeding strategies, and sustainable genetic improvement. The lack of genetic variation resulting from breeding closely related individuals often leads to the expression of genes that are detrimental to reproduction or even survival. This is why intensive selection strategies have drawn the attention of the scientific community; interest exists in preserving, characterizing, and monitoring the autozygosity of important animals for sustainable livestock production [3,4].

Inbreeding is a concerning practice in the livestock industry, considerably influencing genomic patterns, hence designing a tactful breeding scheme is essential. Practically, inbreeding represents the level of homozygosity of a population or within the genome of an individual, which leads to offspring with loss of fitness. The inbreeding coefficient $(F)$ is generally used to estimate the extent of individual's inbreeding, which is traditionally computed from pedigree information. In recent times, there is an increasing interest in estimating the inbreeding coefficient from runs of homozygosity $(\mathrm{ROH})$ as it is likely to be the most powerful method in detecting inbreeding effects from among several alternative estimates. Molecular genetics has facilitated the estimation of the level of autozygosity at individual and population levels resulting from demography, natural and artificial selection, and inbreeding by checking the number of contiguous segments of the genome, referred to as runs of homozygosity (ROH) $[5,6]$. ROH is used to estimate the extent of identical haplotypes in the genome of an individual, transmitted from their parents [2]. A longer $\mathrm{ROH}$ indicates recent inbreeding, whereas a shorter $\mathrm{ROH}$ indicates a loss of genetic diversity resulting from the founder effect or a genetic bottleneck in a population. The selection pressure increase the homozygosity in the targeted genomic region leads to the occurrence of $\mathrm{ROH}$ [7]. Therefore, the genomic regions with high $\mathrm{ROH}$ frequency can be used to detect the association between the genes and traits of interest [8]. Characterization of the homozygosity by descent (HBD) segments associated with $\mathrm{ROH}$, and estimation of the autozygosity in an individual's genome, have become popular techniques as these measures provide insights into the recent history of a population as well as trait architecture [9]. However, until now, the extent of the $\mathrm{ROH}$ across the genome of various goat breeds was poorly understood.

The effective population size $\left(N_{e}\right)$ is the size of a hypothetical ideal population that has the same divergence of gene frequency under random genetic drift or an equal amount of inbreeding as in the real population under consideration [10]. The effective population size is an important parameter for evaluating population genetic diversity and characterizing and understanding the underlying genetic architecture of an animal genome [11]. The $N_{e}$ is crucial in conservation biology as it is used to estimate the rate of genetic drift and inbreeding and affects the systematic evolutionary forces such as selection, mutation, and migration [12]. The $N_{e}$ also provides information on population demographic processes such as migration and admixture, genetic variations, and linkage disequilibrium in a population [11,13].

Reproductive performance is characterized by fecundity, which is crucial to the goat industry. Improvement of reproductive traits, especially the increase in litter size, has attracted widespread interest because small improvements could lead to large gains in profit [14]. With the advancement of molecular genetic technologies, a series of techniques have been developed to identify evidence of selection. Numerous candidate genes and genetic loci have been identified in years using single nucleotide polymorphism (SNP) chips [15]. Due to the availability of the Illumina Goat SNP 50K 
BeadChip (Gene seek/Neogen, San Diego, USA), exploring selection signatures, identifying candidate genes, and genomic diversity assessments resulting from selective pressure have become the main focus of genomic studies in goats.

China is a country with rich and diverse goat genetic resources. About 58 indigenous goat breeds exist in China [16], distributed in different geographical, environmental, and agro-ecological areas, including the north pastoral region, the semi-pastoral-agricultural region, the north and south agricultural regions, and the Qinghai-Tibet plateau region [17]. In the western region of China, the Hengduan Mountains, as a natural geographical barrier, block the Qinghai-Tibet and Yunnan-Guizhou Plateaus, have the most complex river systems (Huaihe River Line), and a profoundly complex and dynamic geological history [18]. The Qinling Mountains, as a vital geographic barrier in Asia, separate the mainland of China into southern semi-tropical and northern temperate regions [19]. These mountains divide Western China into two geographic structures: Northwest China and Southwest China, and are the cause of the different climatic and agro-ecologic characteristics. Since China is a large subcontinent with diverse geographical locations, Chinese goat breeds show tremendous variations in fecundity, production traits, fiber quality, disease resistance, and heat tolerance. Different intensities of natural and artificial selection pressures force them to exhibit different phenotypic characteristics. Among Chinese goat breeds, Jinning Grey (JG), Liaoning cashmere (LN), Guangfeng (GF), Luoping Yellow (LP), Nanjiang (NJ), and Qinggeli (QG) goats are the most popular. Qinggeli and Liaoning Cashmere goats have a relatively low fecundity $(\sim 100 \%)$ compared with Jinning Grey, Nanjiang, Guangfeng, and Luoping Yellow goats ( 200\%). Hence, our goal was to characterize genome-wide $\mathrm{ROH}$ and detection of positive selection signature to provide insights into the patterns of homozygosity, population demographic history, evolutionary mechanisms, and causative genes controlling the phenotypic diversity.

\section{Materials and Methods}

\subsection{Ethics Statement}

Ethical approval for animal survival was provided by the animal ethics committee of the Institute of Animal Science, Chinese Academy of Agricultural Sciences (IAS-CAAS) with the following reference number: IASCAAS-AE-03, on 1 September 2014.

\subsection{Sampling and Genotyping}

A total of 206 goats representing six goat populations, including GF, JG, LN, LP, NJ, and QG, were selected for our study. A short introduction (breed name, sample size, fecundity rate, demography and breeding practices, average altitude and agro-ecology and feeding mode) to these goat breeds is provided in Table S1.

To avoid the probability of relatedness among individuals, samples were collected from different places to represent the within-breed genetic diversity and selection of signature for the reproduction trait. The fecundity rate was obtained from the respective goat farms, where the farmers had recorded it for many years and averaged it per 1000 population for each breed. Then, the fecundity rate was calculated as many offspring can be produced by 100 female goats at one timepoint. We compared the collected fecundity rate with the literature and found similarities [17]. Sample distributions are depicted in Figure 1. Genomic DNA was extracted from blood and tissue samples using the Promega Wizard Genomic Purification Kit (Promega, Madison, USA), according to the standard protocols provided by the manufacturer. Qualified DNA was genotyped using the Illumina Goat SNP 50K Beadchip panel (GeneSeek/Neogen, San Diego, USA). 


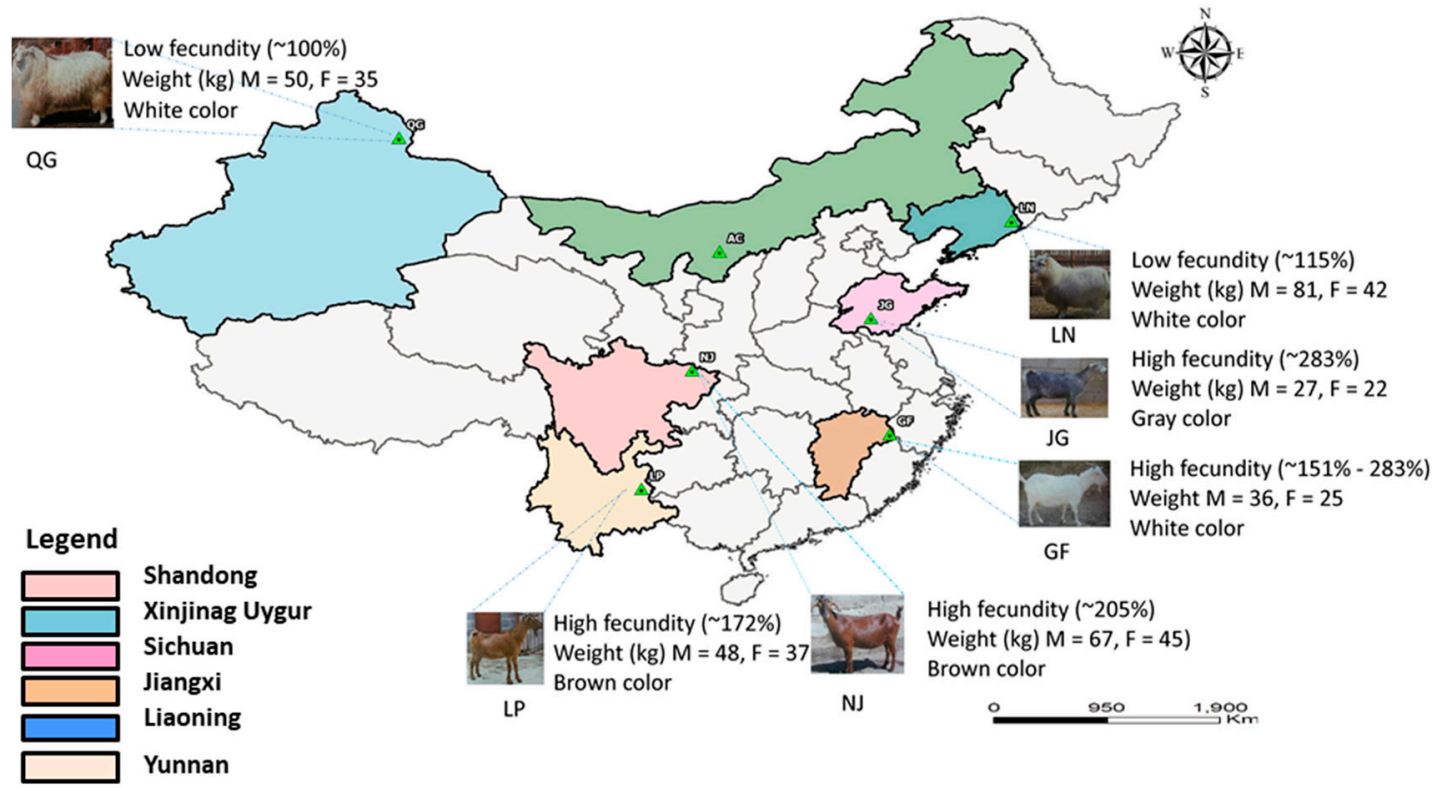

Figure 1. Geographic distribution and phenotypic characteristics of the six goat populations in China. M, Male; F, Female; QG, Qinggeli; LP, Luoping Yellow; NJ, Nanjiang; LN, Liaoning; JG, Jinning Grey; GF, Guangfeng.

\subsection{Data Quality Control}

Quality control of the SNP data was implemented using PLINK v.1.07 [20,21]. The non-informative SNPs that were incompatible with the following criteria were removed from the panel: (1) SNP call rate greater than 95\%, (2) an SNP minor allele frequency greater than 0.05, (3) an SNP with a genotyping rate greater than $95 \%,(4)$ a maximum individual missing genotype rate of more than $10 \%$, or (5) Hardy-Weinberg equilibrium $>1 \times 10^{-5}$.

\subsection{Detection of Runs of Homozygosity}

\subsubsection{Distribution of $\mathrm{ROH}$}

ROHs were computed and characterized for each breed using detectRUNS packages in $R$ software [20]. The following criteria were used to define ROHs: (1) a minimum number of SNPs $=15$, (2) minimum length of $\mathrm{ROH}=1 \mathrm{Mb}$, and (3) one possible heterozygote and one missing genotype were allowed for each $\mathrm{ROH}$ [22]. The number of $\mathrm{ROH}$ s per chromosome was estimated by counting the $\mathrm{ROH}$ number for each chromosome. The percentage of the chromosome covered by ROHs was calculated by the formula suggested by Al-Mamun et al. [23]: summing all the ROHs on a chromosome divided by the number of individuals containing $\mathrm{ROHs}$ on that chromosome produces the mean $\mathrm{ROH}$ length; the mean ROHs length of that chromosome is then divided by the respective chromosome length and multiplied by 100 to convert into a percentage. The total number, length of $\mathrm{ROH}$ (in Megabases), and the sum of all $\mathrm{ROH}$ segments (in Megabases) were calculated for each breed. We categorized the $\mathrm{ROH}$ lengths into six length classes $(0-3,3-5,5-10,10-20,20-30$, and $>30 \mathrm{Mb})$ to compare the distribution of $\mathrm{ROH}$ length in each breed. We calculated the frequency and average $\mathrm{ROH}$ length for each length category. The average $\mathrm{ROH}$ length for each class of each breed was estimated by summing all $\mathrm{ROH}$ segments of each ROH length class per breed and dividing by the total number of individuals of that respective breed. 


\subsubsection{Inbreeding Coefficient}

The genomic inbreeding coefficient $\left(F_{\mathrm{ROH}}\right)$ for each breed was computed using the following method [24]:

$$
F_{\mathrm{ROH}}=\left(\mathrm{L}_{\mathrm{ROH}} / \mathrm{L}_{\mathrm{AUTO}}\right),
$$

where $\mathrm{L}_{\mathrm{ROH}}$ is the total length of $\mathrm{ROH}$ of each individual in the genome and $\mathrm{L}_{\mathrm{AUTO}}$ is the length of the autosomal genome of the goat (set to $2399.4 \mathrm{Mb}$ [22]). To verify the accuracy of $F_{\mathrm{ROH}}$, we also calculated the inbreeding coefficient based on the difference between the expected and observed numbers of homozygous genotypes $\left(F_{\mathrm{HOM}}\right)$ with the command-het using PLINK v.1.07. The correlation between $F_{\mathrm{ROH}}$ and $F_{\mathrm{HOM}}$ was calculated across the breeds.

\subsubsection{Detection of Common ROHs}

To identify the genomic regions with a high $\mathrm{ROH}$ frequency, the percentage of occurrences of an SNP in an ROH was calculated by counting the number of times the SNP was detected in an ROH across the population. The SNPs showing a percentage higher than $45 \%$ were selected as genomic regions with a high frequency of $\mathrm{ROH}$ s for further analysis. We used the caprine reference genome annotation file from the NCBI website to annotate the genes identified at particular genome coordinates for all the selected regions [25].

\subsection{Homozygosity by Descent (HBD)}

A hidden Markov model (HMM)-based approach was implemented in R CRAN: (https://CRAN. Rproject.org/package $=\mathrm{RZooRoH}$ ) to scan the individual genome for the HBD segments, as described in Solé and Gori [26] and Bertrand and Kadri [9]. A MixKR model was applicable, where nine HBD states with respective rates $\left(R_{k}\right)$ of $\left(2^{1}, 2^{2}, 2^{3}, \ldots, 2^{9}\right)$ and one non-HBD with an $R_{k}$ rate of $2^{9}$ were used. The number of classes, $K$, was fixed for each run. Only one non-HBD class and $K-1$ HBD class for a total of $K=10$ class were considered. The predefined rate of $K-1 \mathrm{HBD}$ classes always ranged 2 to $2^{K-1}$, whereas the rate of the non-HBD class was fixed as the most ancient class. Each $K$ has its own rate parameter, $R_{K}$, which indicates the lengths of the segments for its respective class. The length of HBD classes is exponentially distributed with rate $R_{k}$, which is double the number of generations to the common ancestor of the respective class, and this is called a K.R model [26]. The length of the HBD segment is $1 / R$ Morgans, indicating high rates associated with shorter segments. To estimate the inbreeding coefficient, we considered the ancestors with an $R_{k}$ rate higher than threshold $T$ as unrelated. The corresponding genomic inbreeding coefficient $\left(F_{G-T}\right)$ was then estimated, with $R_{K} \leq T$ averaged over the whole genome.

\subsection{Effective Population Size $\left(N_{e}\right)$}

Effective population size $\left(N_{e}\right)$ was calculated for each breed separately using SNeP v1.1 [27]. $N_{e}$ estimates at different time points are based on linkage disequilibrium, using the formula proposed by Sved [28]:

$$
E\left(r^{2}\right)=\frac{1}{1+4 N_{e} c},
$$

where $N_{e}$ is the effective population size, $c$ is the genetic distance in Morgans, and $E\left(\mathrm{r}^{2}\right)$ is the expected average coefficient of determination $\left(r^{2}\right)$ value for distance $c$. Each time point corresponding to a genetic distance was calculated as: $T=\frac{1}{2 c}$. Estimated effective population sizes $\left(N_{e}\right)$ against their past generations were plotted in $\mathrm{R}$ over the last 100 and 1000 generations.

\subsection{Population Differentiation, Analysis of Molecular Variance, and Structure}

Reynolds's genetic distance and pairwise difference were computed using the integrated software for a different hierarchical level of population genetic data analysis, using ARLEQUIN v.3.5.2 [29] with 
100 permutations and a significance level of 0.05 . The analyses of molecular variance (AMOVA) to test the partition of genetic diversity were performed using ARLEQUIN v.3.5.2.

\subsubsection{Principal Component Analysis}

To investigate the genetic relationship between individuals, we used the principal component analysis (PCA). A pruned set of SNPs that were in approximate linkage equilibrium with each other were used to avert clustering [30]. The PLINK indep-pairwise option (indep-pairwise 50, 10, 0.1), where the command considers each window of 50 SNPs, was used to find the pruned set of SNPs [20]. The criterion of a pairwise linkage disequilibrium $\left(r^{2}\right)$ value of $<0.1$ was used to obtain the SNP panel that approximates linkage equilibrium. In this study, PCA was performed using PLINK software (parameter: pca) and the output was visualized using R software.

\subsubsection{Neighbor-Joining Tree}

To further confirm the phylogenetic relationships among the breeds, we constructed a neighbor-joining (NJ) tree using MEGA v.5.0 [31]. We calculated the genetic distance matrix using PLINK v.1.09 $[20,21]$ (parameter: distance-matrix) and then, based on this distance matrix, we constructed the Neighbor-joining tree using MEGA v.5.0.

\subsubsection{Admixture Analysis}

To examine the pattern of genetic variation among the breeds, a model-based clustering approach was applied using the ADMIXTURE program v.1.2 [32]. The ancestral source $\mathrm{K}$ refers to the number of populations used in this dataset. A five-fold cross validation (CV) error for each $\mathrm{K}$ was used to select the best $\mathrm{K}$.

\subsection{Selective Sweep Analysis}

We used the population differentiation index $\left(F_{\mathrm{ST}}\right)$, nucleotide diversity $(\pi)$, and cross-population extended haplotype homozygosity (XP-EHH) approach to identify the genomic regions that appear to be the target of selection. We categorized the goat breeds into high and low groups according to their reproductive performance, and compared the high fecundity $(\sim 200 \%)$ goat breeds group (JG, GF, NJ, and LP) with the low fecundity ( 100\%) goat breeds (LN and QG) to explore positive selection signatures.

\subsubsection{Population Differentiation Index $\left(F_{\mathrm{ST}}\right)$ Approach}

The population differentiation index $F_{\mathrm{ST}}$ is an important indicator for testing the footprint of positive selection and elicit whether genetic differentiation exists between populations. The unbiased metric of pairwise $F_{\mathrm{ST}}$ values were computed, as described by Weir et al. [33]. We then transformed $Z$ into $F_{\mathrm{ST}}$ values [34].

\subsubsection{Nucleotide Diversity $(\pi)$}

Another popular approach to calculate the degree of polymorphism within a population is nucleotide diversity $(\pi)$. We calculated the site nucleotide diversity $(\pi)$ as the proportion of pairwise differences between two populations, ( $\pi$ (low fecundity goat)/ $\pi$ (high fecundity goat)) to investigate the genetic variation between the groups.

\subsubsection{Estimation of Cross-population extended haplotype homozygosity (XP-EHH)}

The XP-EHH estimate is often used to detect a positive selection event, mainly to compare the haplotype homozygosity (EHH) and integrated haplotype score (iHS) between two populations. The basic principle of XP-EHH estimation involves scanning the distinct SNPs between populations that are homozygous for one and polymorphic for others through the comparison of the EHH score of 
two populations. A positive $\mathrm{XP}-\mathrm{EHH}$ value indicates selection occurs in the test population; a negative value indicates selection in the control population. The calculation formula is

$$
X P-E H H=\ln \left(\frac{I_{A}}{I_{B}}\right),
$$

where $I_{A}$ is the integrated value of the test population $\mathrm{EHH}$ and $I_{B}$ is the integrated value of the reference population $\mathrm{EHH}$. The codes from the following website were used to calculate the XP-EHH: http://hgdp.uchicago.edu/software/xpehh.tar. The calculated raw XP-EHH statistics were then standardized to a distribution with zero mean and unit variance.

The regions showing extremely high $F_{\mathrm{ST}}$ values and elevated nucleotide diversity (top $1 \%$ of both) were considered to be potentially selected candidate outliers under selection. We also scanned the top $0.1 \%$ of XP-EHH estimates to improve the confidence in the selected outliers under selection.

\subsubsection{Gene Annotation and Functional Enrichment Analysis}

Based on the above findings, the cross top $1 \%$ values of $F_{\mathrm{ST}}$ and $\pi$ and $0.1 \%$ of XP-EHH were considered as selective signals and were extended up to $100 \mathrm{~kb}$ upstream and downstream to cover the candidate region. To annotate the candidate region, we downloaded the caprine gene from Ensemble (http://www.ensemble.org/) and annotated it using R v.3.5.1. The overlapping genes retrieved from the two analytical approaches were used for enrichment analysis. Gene ontology (GO) and Kyoto Encyclopedia of Genes and Genomes (KEGG) pathway analysis of the target genes were performed using a web-based toolset: g: profiler (https://biit.cs.ut.ee/gprofiler/gost) and KOBAS v.3.0 (http://kobas.cbi.pku.edu.cn/anno_iden.php), respectively. As only limited genes are annotated in the goat genomes, we used the human genome as the background for enrichment analysis.

\section{Results}

\subsection{Sample and SNP Filtration}

From the 206 individuals tested, only two individuals (one from JG and another from LN) were removed from further analysis due to their low genotyping rate (Maximum individual missingness rate $>0.1$ ). The total genotyping rate in the remaining individuals was 0.98147 . A total of 1338 SNPs with a call rate $<0.95,2296$ SNPs with a minor allele frequency $<0.05$, and 1814 SNPs with a Hardy-Weinberg equilibrium $\left(p<1 \times 10^{-5}\right)$ were excluded from the panel (Table 1). After the quality filtration and exclusion of SNPs from the sex chromosome, 45,311 SNPs from 204 individuals were used for downstream analyses.

Table 1. Single nucleotide polymorphisms (SNPs) filtration result.

\begin{tabular}{cc}
\hline Parameters & Goat 50K \\
\hline Total number of SNPs & 51,474 \\
SNP Call frequency (call rate) $(<0.95)$ & 1338 \\
Minor allele frequency $(<0.05)$ & 2296 \\
Hardy-Weinberg equilibrium $\left(p<1 \times 10^{-5}\right)$ & 1814 \\
SNPs removed from sex chromosome & 715 \\
Total SNPs removed & 6163 \\
Total SNPs remained & 45,311 \\
\hline
\end{tabular}

\section{2. $\mathrm{ROHs}$}

\subsection{1. $\mathrm{ROH}$ Patterns}

ROHs, representing the level of genomic autozygosity, are continuous homozygous segments at the individual and population levels that can be used as a measurement of inbreeding; more in-depth 
ROHs are the result of demography, natural and artificial selection, and inbreeding [6,35]. According to the parameters set, $18,066 \mathrm{ROHs}$ were found in total, with a mean of 88.55 per individual, ranging from 11 (QG) to $244(\mathrm{GF})$. LN presented the largest number of ROHs $(n=7425)$, followed by GF $(n=3415)$. GF displayed the longest ROHs on average (mean $=3.29 \pm 0.001 \mathrm{Mb})$ and the shortest average $\mathrm{ROH}$ length was found in LP $(1.95 \pm 0.001 \mathrm{Mb})$. Among all the ROHs, the longest ROH was detected on chromosome 20 of the LP goat breed $(67.54 \mathrm{Mb})$, consisting of 1304 SNPs (Table 2). A total of $812 \mathrm{ROHs}$ were longer than $10 \mathrm{Mb}$ (225 for GF, 78 for JG, 375 for LN, 80 for LP, 33 for NJ, and 21 for QG). Chromosome 1 exhibited the maximum number of ROHs $(n=1027)$, followed by chromosome 2 $(n=920)$ (Figure 2). The total number of ROHs per chromosome tended to decrease with decreasing chromosome length. The highest and lowest percentages of ROHs per chromosome were calculated for chromosome $28(6.23 \%)$ and chromosome $1(1.72 \%)$, respectively (Figure 2).

Table 2. Descriptive statistics for runs of homozygosity and inbreeding coefficients (F) within each breed. SE, standard error; $\boldsymbol{F}_{\mathbf{R O H}}$, inbreeding coefficient based on runs of homozygosity $(\mathrm{ROH})$; $\boldsymbol{F}_{\text {HOM, }}$ Inbreeding coefficient based on the difference between the observed and expected numbers of homozygous genotypes; $R$, correlation; GF, Guangfeng; JG, Jinning Grey; LN, Liaoning; LP, Luoping Yellow; NJ, Nanjiang; and QG, Qinggeli.

\begin{tabular}{|c|c|c|c|c|c|c|c|c|c|}
\hline \multirow{2}{*}{ Breed } & \multirow{2}{*}{ SNP No. } & \multicolumn{2}{|c|}{ Average Length $(\mathrm{Mb})$} & \multicolumn{2}{|c|}{ Average Number } & \multicolumn{2}{|c|}{$F_{\mathrm{ROH}}$} & \multirow[b]{2}{*}{ Mean \pm SE } & \multirow{2}{*}{$\begin{array}{c}R \\
\left(F_{\mathrm{ROH}},\right. \\
\left.F_{\text {HOM }}\right)\end{array}$} \\
\hline & & Mean \pm SE & Range & Mean \pm SE & Range & Mean \pm SE & Range & & \\
\hline GF & $15-1195$ & $3.29 \pm 0.001$ & $1.00-64.21$ & $142.29 \pm 2.41$ & $53-244$ & $0.19 \pm 0.005$ & $0.05-0.48$ & $0.057 \pm 0.007$ & 0.97 \\
\hline JG & 15-1192 & $2.5 \pm 0.002$ & $1.00-61.64$ & $35.50 \pm 0.23$ & $13-76$ & $0.03 \pm 0.009$ & $0.006-0.24$ & $-0.002 \pm 0.001$ & 0.89 \\
\hline LN & $15-1066$ & $2.99 \pm 0.000$ & $1.00-55.87$ & $130.26 \pm 0.21$ & $91-162$ & $0.162 \pm 0.0004$ & $0.105-0.234$ & $-0.034 \pm 0.001$ & 0.96 \\
\hline LP & $15-1304$ & $1.95 \pm 0.001$ & $1.00-67.54$ & $129 \pm 0.82$ & $67-154$ & $0.105 \pm 0.002$ & $0.03-0.29$ & $0.010 \pm 0.003$ & 0.95 \\
\hline NJ & $15-580$ & $2.59 \pm 0.001$ & $1.16-30.61$ & $69.79 \pm 0.63$ & $26-86$ & $0.06 \pm 0.0009$ & $0.013-0.117$ & $-0.020 \pm 0.001$ & 0.98 \\
\hline QG & $15-1158$ & $2.34 \pm 0.006$ & $1.00-61.65$ & $26.83 \pm 0.45$ & $11-61$ & $0.026 \pm 0.001$ & $0.006-0.225$ & $-0.008 \pm 0.001$ & 0.99 \\
\hline
\end{tabular}

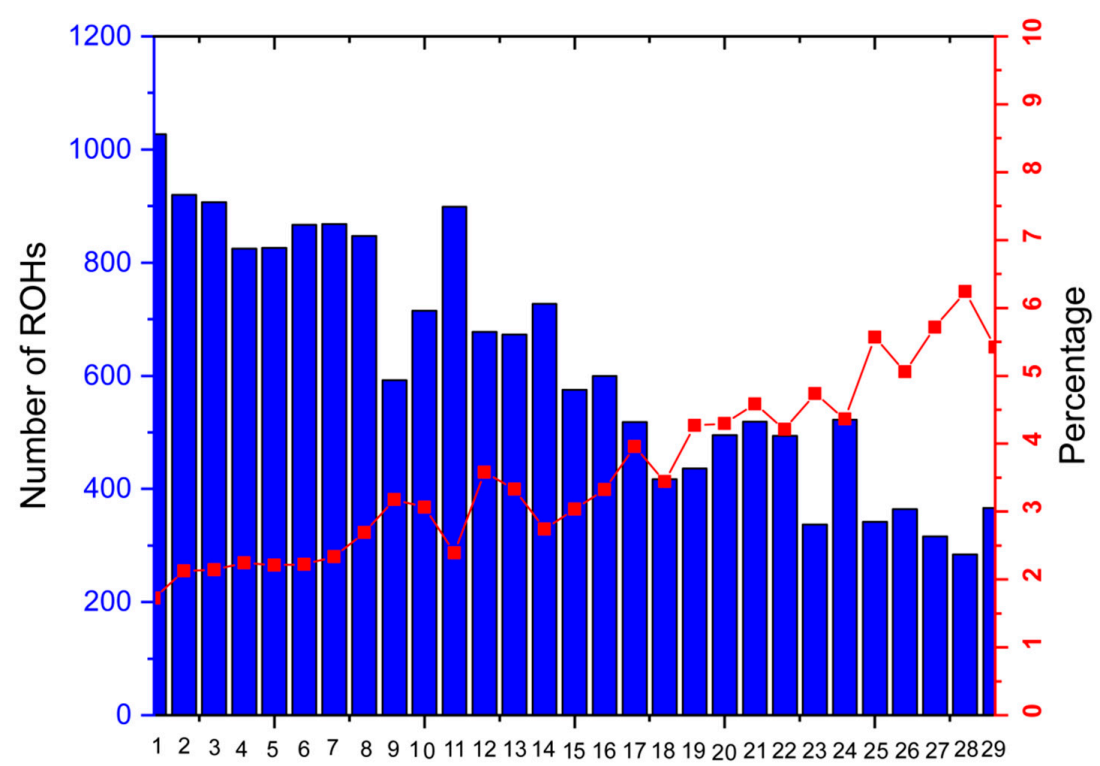

Chromosome

Figure 2. Number of runs of homozygosity (ROHs) per chromosome identified in 204 goat breeds that had at least one $\mathrm{ROH}$ (bars), and the percentage of each chromosome covered by ROHs (lines).

Figure 3 shows the relationship between the number of ROHs and the total genomic length covered by ROHs per individual, which varies considerably among breeds. The QG breed presented with a large number of short ROHs relative to the other breeds, and the GF goat displayed some extreme individuals with $\mathrm{ROH}$ coverage of more than $600 \mathrm{Mb}$ (Figure 3). 


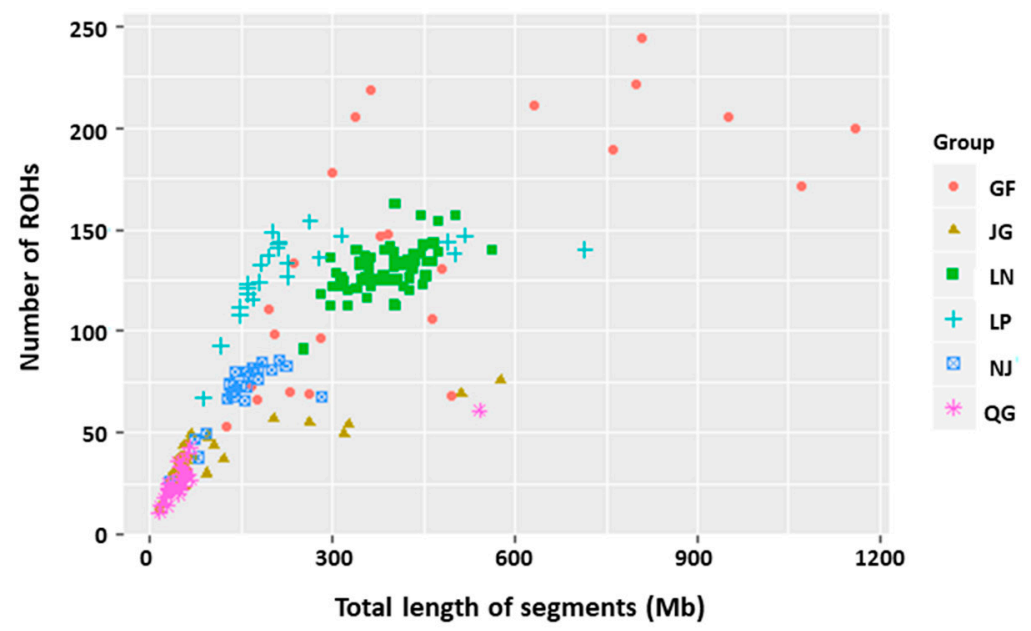

Figure 3. Total genomic length (Megabases) covered by ROH per individual ( $x$ axis) and total number of $\mathrm{ROH}$ per individual ( $y$ axis).

The frequencies of $\mathrm{ROH}$ numbers in different length classes are illustrated in Figure 4A. Our results show an inverse relationship between $\mathrm{ROH}$ length and frequency. The frequencies of shorter $\mathrm{ROHs}$ (0-3) predominated; the number of these ROHs accounted for $82.72 \%$ of the total number of ROHs. However, the frequency of $\mathrm{ROHs}$ in this major class varied between breeds. The majority, more than $90 \%$ of the ROHs in this class, were obtained from LP breeds, whereas the percentage was at least $80 \%$ for GF, JG, NJ, and QG, and less than 80\% for the LN breed. GF and LN had the highest frequencies of ROHs among all breeds in the length category greater than three. Figure $4 \mathrm{~B}$ shows the distribution of $\mathrm{ROH}$ within breeds. Among the six $\mathrm{ROH}$ categories under consideration, the short $\mathrm{ROHs}(<3)$ are most prevalent across the populations, wherein the average ROH mean spanned from 31 (QG) to 175 (GF). The longest $\mathrm{ROH}$ category ( $>30$ ) was the rarest, wherein the GF breed displayed the highest mean $\mathrm{ROH}$ compared with the other breeds.

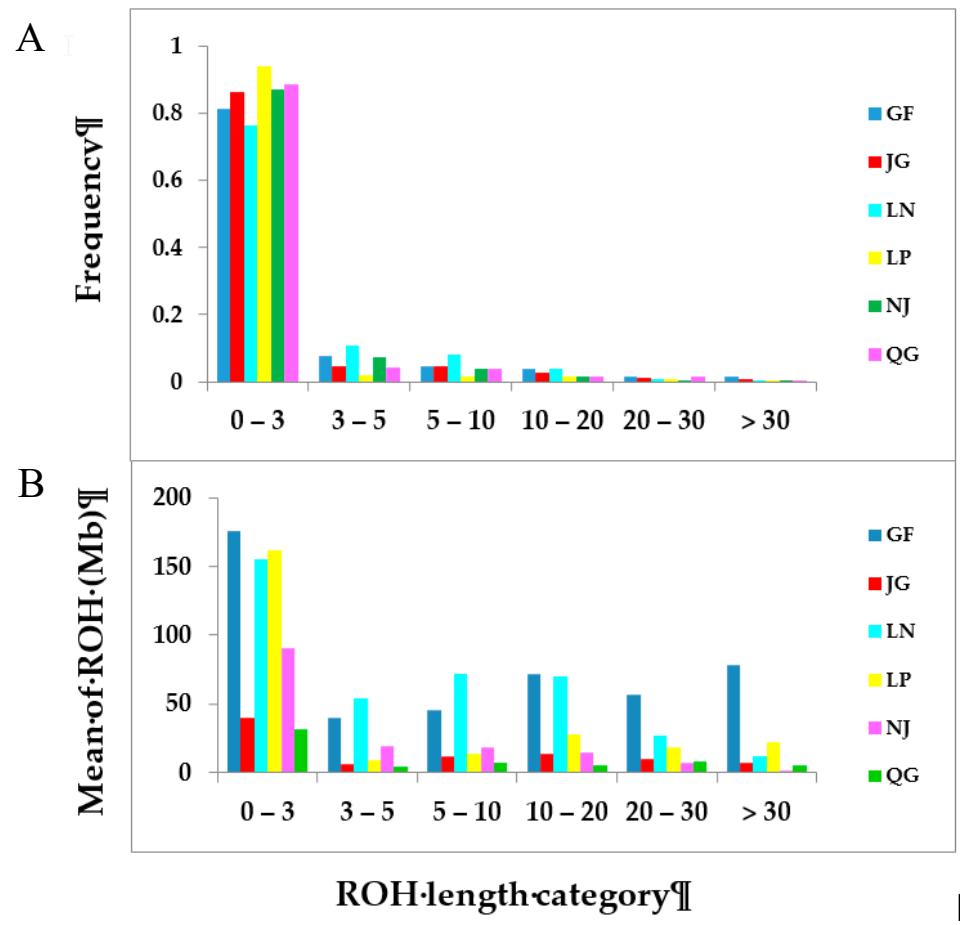

Figure 4. (A) Frequency of ROHs in different length categories in six goat populations. (B) The average sum of runs of homozygosity $(\mathrm{ROH})$ of each breed in different $\mathrm{ROH}$ length classes. 
To investigate the $\mathrm{ROH}$ content in each breed, we also calculated the sum of all $\mathrm{ROH}$ lengths for each individual within the breeds and found that the LN had more ROHs than others (Figure 5). Individuals of the LN breed generally exhibited the highest average sum of ROH content $(256.03 \mathrm{Mb})$ on their genome, followed by GF (117.75 Mb) and LP (106.75 Mb) (Figure 5, Table S2). In contrast, the QG individuals displayed the lowest average sum of $\mathrm{ROH}(22.20 \mathrm{Mb})$.

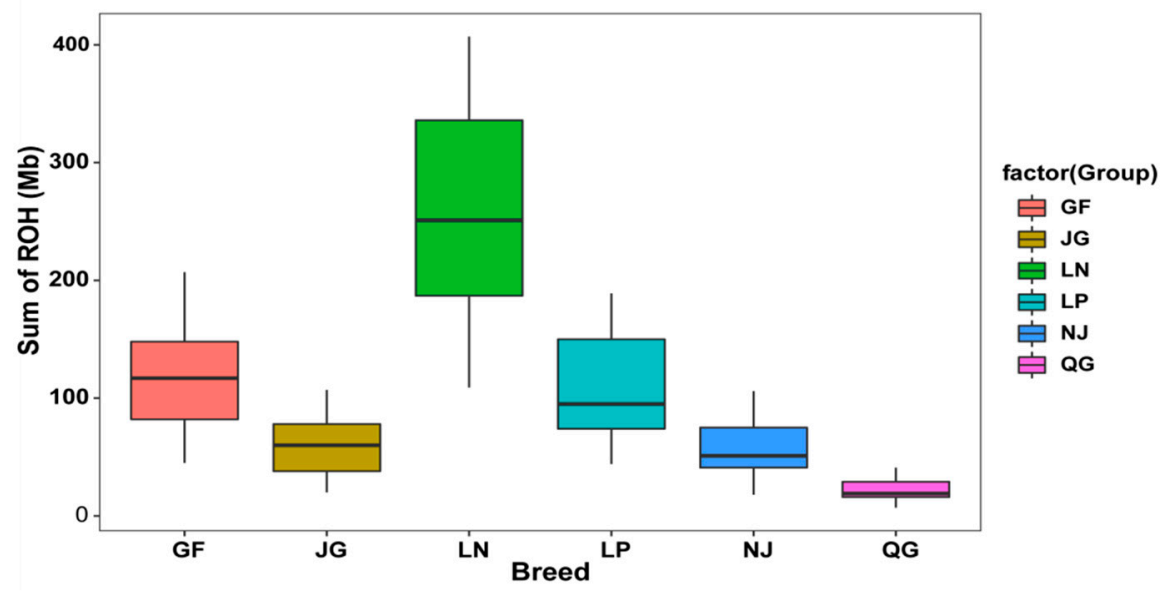

Figure 5. The sum of $\mathrm{ROH}$ length $(\mathrm{Mb})$ per individual genome in each breed.

\subsubsection{Inbreeding Coefficient of Runs of Homozygosity $\left(F_{\mathrm{ROH}}\right)$}

The mean inbreeding coefficient estimates, with their range in variation and distribution in the studied goat breeds, are reported in Table 2 and Figure 6 . The mean $F_{\mathrm{ROH}}$ for all breeds ranged from 0.026 (QG) to 0.19 (GF) (Table 2). The GF goat is the most inbred goat, followed by LN (0.162) and LP (0.105). At the individual level, the individuals of the GF breed displayed the highest $F_{\mathrm{ROH}}(0.48)$, including some individuals with extreme values compared with other breeds.

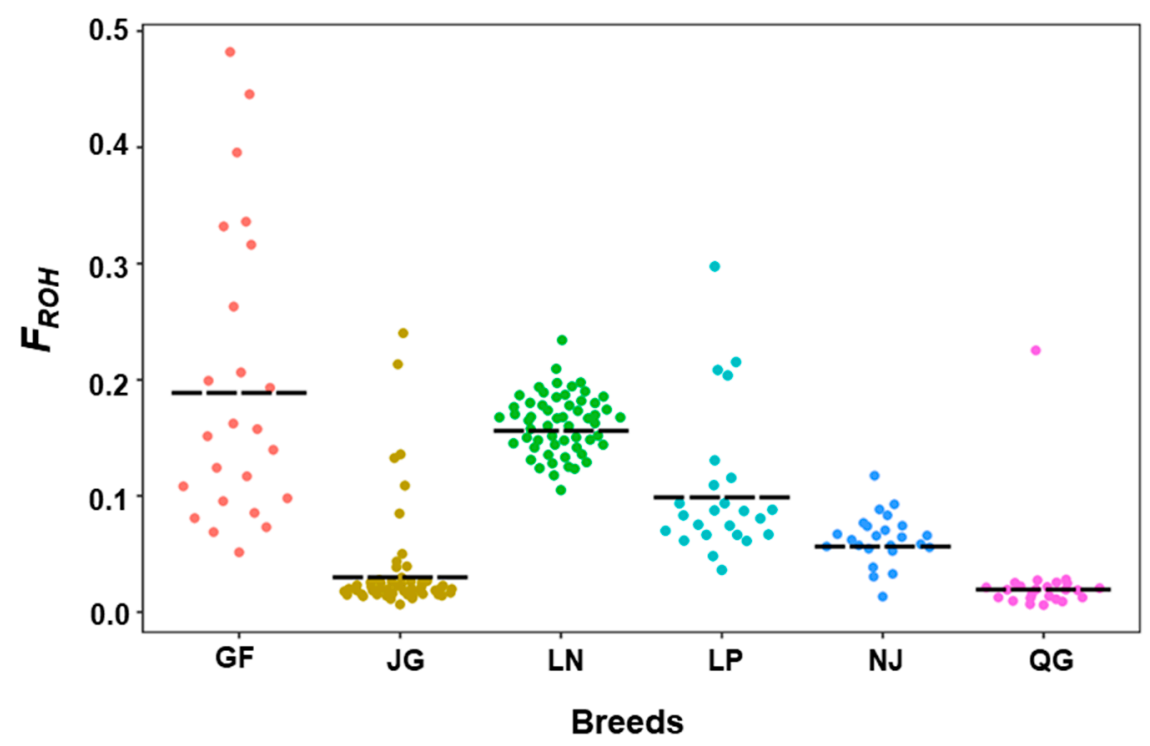

Figure 6. Distribution of the runs of homozygosity inbreeding coefficient $\left(F_{R O H}\right)$ within each breed.

The $F_{\mathrm{HOM}}$ results indicated positive values only for the GF and LP breeds. The $F_{\mathrm{ROH}}$ was higher in GF in both the short- and long-length classes, whereas LN showed a higher $F_{\mathrm{ROH}}$ in the medium-length class (Table 3). The correlations between $F_{\mathrm{ROH}}$ and $F_{\mathrm{HOM}}$ were found to be highest for QG (0.99), followed by NJ (0.98) and GF (0.97) (Table 2). The $F_{\mathrm{ROH}}$ was higher in GF in most of the ROH length classes, with a higher mean FROH across the classes (Table 3). 
Table 3. Estimated inbreeding levels at each cut-off point of runs of homozygosity.

\begin{tabular}{cccccccc}
\hline Breed & $\boldsymbol{F}_{\text {ROH (0-3) }}$ & $\boldsymbol{F}_{\text {ROH (3-5) }}$ & $\boldsymbol{F}_{\text {ROH (5-10) }}$ & $\boldsymbol{F}_{\text {ROH (10-20) }}$ & $\boldsymbol{F}_{\text {ROH (20-30) }}$ & $\boldsymbol{F}_{\text {ROH (>30) }}$ & Mean \\
\hline GF & 0.060167 & 0.013693 & 0.015554 & 0.024719 & 0.019603 & 0.026762 & 0.0267 \\
JG & 0.013824 & 0.002124 & 0.003958 & 0.004607 & 0.003361 & 0.002482 & 0.0050 \\
LN & 0.053225 & 0.018406 & 0.024548 & 0.02398 & 0.009217 & 0.004083 & 0.0222 \\
LP & 0.055294 & 0.003027 & 0.004658 & 0.009596 & 0.006219 & 0.007642 & 0.0144 \\
NJ & 0.030963 & 0.006652 & 0.006211 & 0.005015 & 0.002545 & 0.000437 & 0.0086 \\
QG & 0.010724 & 0.001535 & 0.002563 & 0.001888 & 0.002893 & 0.001974 & 0.0035 \\
\hline
\end{tabular}

\subsubsection{Genomic Regions with a High ROH Frequency}

The most common genomic regions associated with $\mathrm{ROHs}$ in the six goat populations were detected, and the percentage of SNPs in ROHs were assessed by calculating the frequency of SNPs occurring in those ROHs across individuals. The output was plotted against the position of the SNP along the chromosome (Figure 7). A total of 97 genomic regions were identified (Table S3). Here, we focused on the selected regions and investigated if the identified regions coincided with the regions harboring the genes involved in reproduction. We identified six genes in the selected $\mathrm{ROH}$ regions that are associated with reproduction. We found that the ROHs on chromosomes 25 and 13 overlapped with regions detected by the selection signature, which spanned MARF1 and SYCP2, respectively. Another two regions on chromosomes 4 and 24 were near to the region detected by the selection signature, which contain $A D C Y 1$ and TMEM200C, respectively; these genes are also associated with reproductive processes.

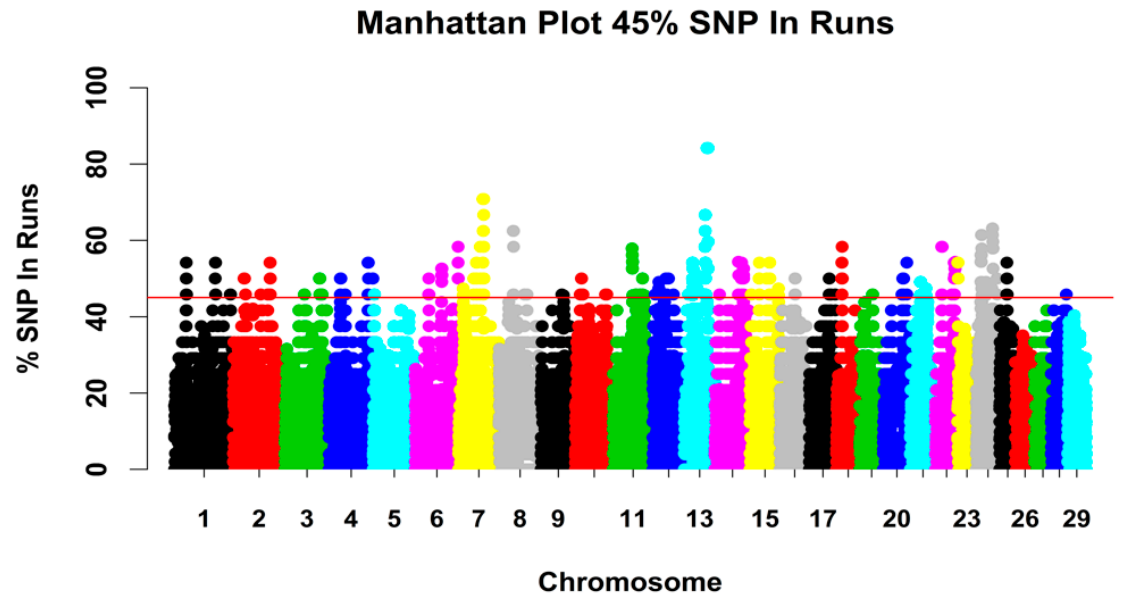

Figure 7. Manhattan plot of the occurrences (\%) of an SNP in ROHs across the population. A dot represents an SNP.

\subsection{Homozygosity by Descent (HBD) Classes}

The inheritance of two copies of the same chromosomal segments in an individual's genome from an ancestor tracing back to different periods is referred to as homozygosity by descent (HBD) segments. The variable sizes of these segments are associated with the different ancestors that can be traced back to different generations in the past. The rate of the HBD class is inversely associated with the size of the segments. Long HBD segments correspond to recent common ancestors, whereas short segments indicate autozygosity inherited from ancient common ancestors.

Figure 8 presents the partitioning of individual genomes in seven different HBD classes. A difference was observed in terms of partitioning. The individuals from JG and QG showed a limited amount of autozygosity associated with small and ancient HBD segments (indicated in blue). The GF breed displayed more variation in autozygosity and length of HBD segments. The most inbred GF population exhibited an autozygosity level of more than 0.4, mainly associated with a distant 
ancestor, with indications that the ancestors contributed traces back 128 generations. Some individuals of GF exhibited HBD segments from recent common ancestors. LN individuals showed a higher level of autozygosity compare with the LP and NJ breeds, but this is associated with more distant ancestors (with the HBD classes with ratings of 16 to 128 ).

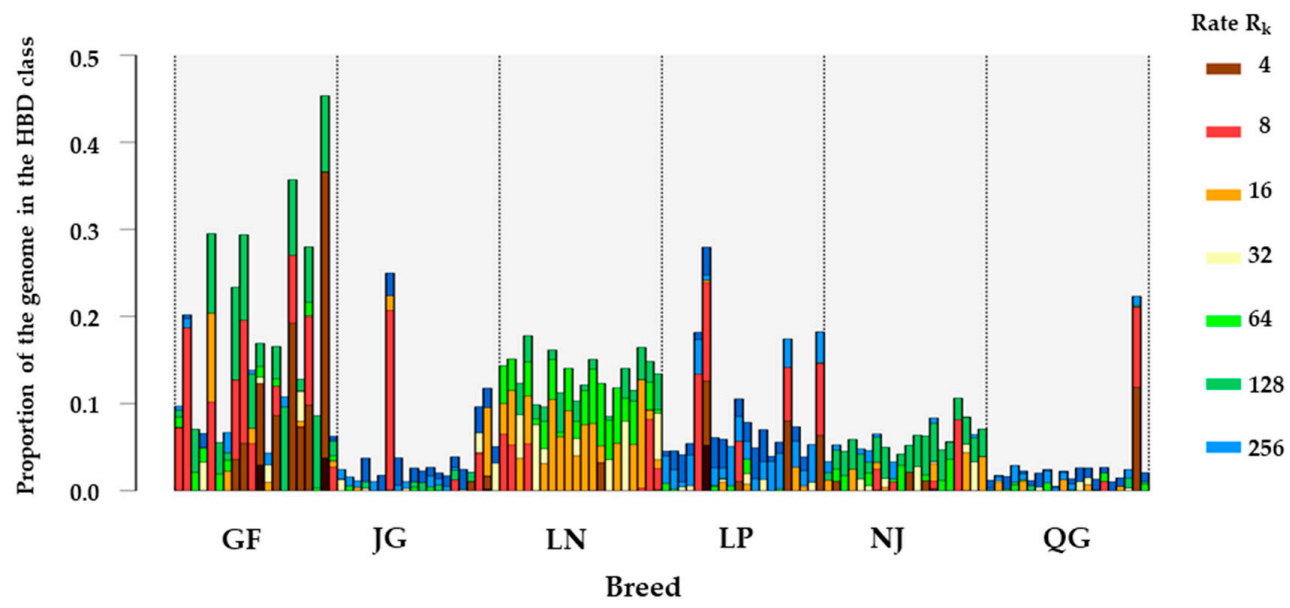

Figure 8. Partitioning of the genome in different homozygosity by descent (HBD) classes in six goat populations. The results are plotted for 20 randomly selected individuals per population. The height of each bar represents the proportion of the genome associated with the HBD class of the corresponding color.

The average inbreeding coefficient was estimated using the HBD class. GF goats displayed the highest average inbreeding coefficient, followed by the LN breed. An increasing trend of inbreeding coefficient with ancient classes was observed across the breeds, whereas the GF goats showed a rapid increase in the inbreeding coefficient from 0.00 for an estimate based on the first class $\left(R_{K}=2\right)$ to 0.09 with $R_{K} \leq 8$, and then increasing marginally up to $R_{K} \leq 64$. The highest value was observed at $\mathrm{R}_{\mathrm{K}}=512$ (0.169) (Figure 9). A similar pattern for the inbreeding coefficient was found for LN: rapidly increasing up to $R_{K} \leq 64$, and the highest inbreeding coefficient was found at $R_{K} \leq 128(0.134)$.

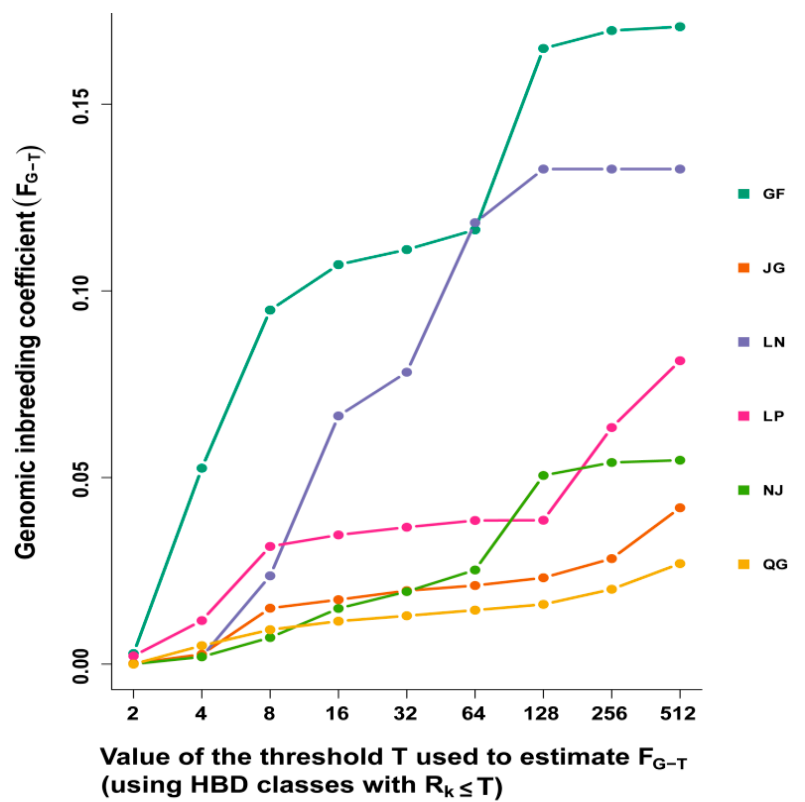

Figure 9. Value of the threshold $T$ used to estimate $F_{G-T}$ (using HBD classes with $R_{K} \leq T$ ). 


\subsection{Effective Population Size $\left(N_{e}\right)$}

Ancestral and recent effective population sizes $\left(N_{e}\right)$ for six goat populations are presented in Figure 10. Estimated $N_{e}$ showed a downward trend with the increase in generations across the populations. The most rapidly declining recent $N_{e}$ was found in the GF and LN breeds, whereas JG and QG showed a slowly declining trend in $N_{e}$ (Figure 10b).

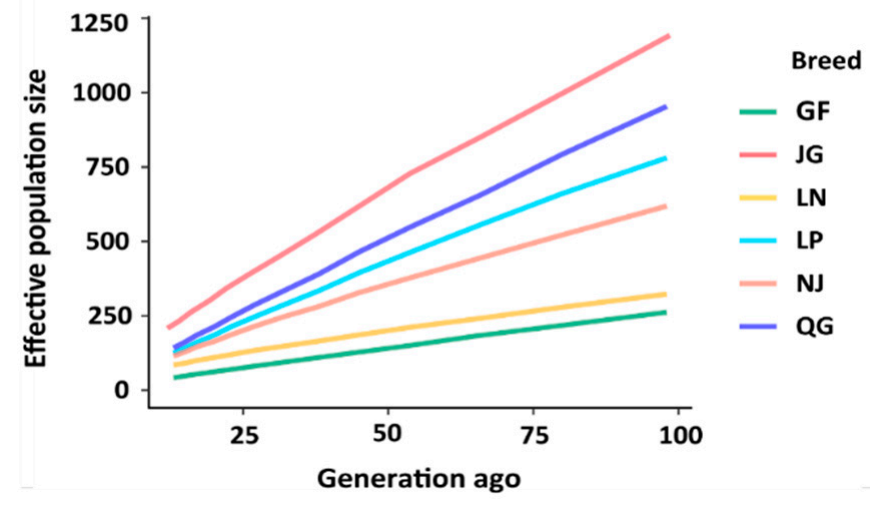

(a)

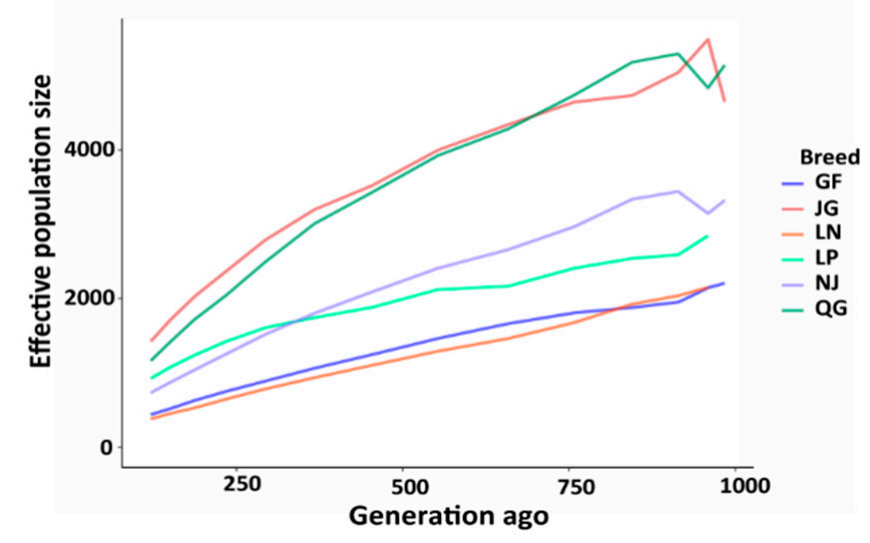

(b)

Figure 10. Estimated effective population sizes $\left(N_{e}\right)$ in six Chinese goat populations. (a) $N_{e}$ in the past 100 generations. (b) $N_{e}$ over the past 1000 generations.

\subsection{Population Differentiation, AMOVA, and Structure}

Population genetic differentiation was evaluated by calculating Reynolds' genetic distance and pairwise differences. Our result showed that the Reynolds' genetic distance $\left(D_{R}\right)$ values vary from 0.02116 (NJ-QG) to 0.20055 (LN-LP), whereas the highest and lowest pairwise differences were found for LN-LP (0.18172) and NJ-QG (0.02099), respectively (Table 4).

Table 4. Reynolds' genetic distances (above diagonal) and pairwise differences (below diagonal) among four Chinese goat populations.

\begin{tabular}{ccccccc}
\hline Breed & GF & JG & LN & LP & NJ & QG \\
\hline GF & & 0.06847 & 0.17687 & 0.17422 & 0.11081 & 0.08963 \\
JG & 0.06618 & & 0.11153 & 0.11506 & 0.04969 & 0.02867 \\
LN & 0.16211 & 0.10554 & & 0.20055 & 0.06391 & 0.08185 \\
LP & 0.15989 & 0.10869 & 0.18172 & & 0.13337 & 0.10824 \\
NJ & 0.10489 & 0.04848 & 0.06191 & 0.12486 & & 0.02116 \\
QG & 0.08573 & 0.02826 & 0.07859 & 0.10259 & 0.02094 & \\
\hline
\end{tabular}


Analysis of molecular variance (AMOVA) illustrated that intra-population variation accounted for $89.61 \%$ of the total variation, with $9.68 \%(p<0.001)$ of the variation being inter-population (Table S4).

\subsection{Detection of Signature of Selection}

\subsubsection{Distinct Population Structure Pattern}

The PCA results showed that the first principal component (PC1) accounted for $18.09 \%$ of the genetic variation, resulting in the clear segregation of LN and a clear separation of the LP breed in the positive direction of the second principal component (PC2), which explained $12.50 \%$ of the total variance (Figure 11A). The NJ breed is clustered together with QG and a degree of overlap was detected between JG and GF, which is in accordance with their geographic distribution in China (Figure 1). This finding is consistent with the result of the NJ tree, wherein the LN and LP breeds formed genetically distinct groups (Figure 11B). The NJ and QG goat breeds are clustered together and the degree of differentiation was low between JG and GF in the NJ tree analysis (Figure 11B). To further verify the PCA results, we performed population admixture analysis, where the least amount of cross-validation error occurred when $\mathrm{K}=5$ (Figure $11 \mathrm{C}$ ), which indicates that $\mathrm{K}=5$ was the optimal modeling choice. Our admixture result showed that the LN and LP goat breeds displayed a separate group when $\mathrm{K}=3$. However, the QG admixed with NJ and JG, and GF goat breeds are mixed with each other (Figure 11D) when $\mathrm{K}=4$ or 5 . The results of the admixture were consistent with the results of PCA and the NJ tree.
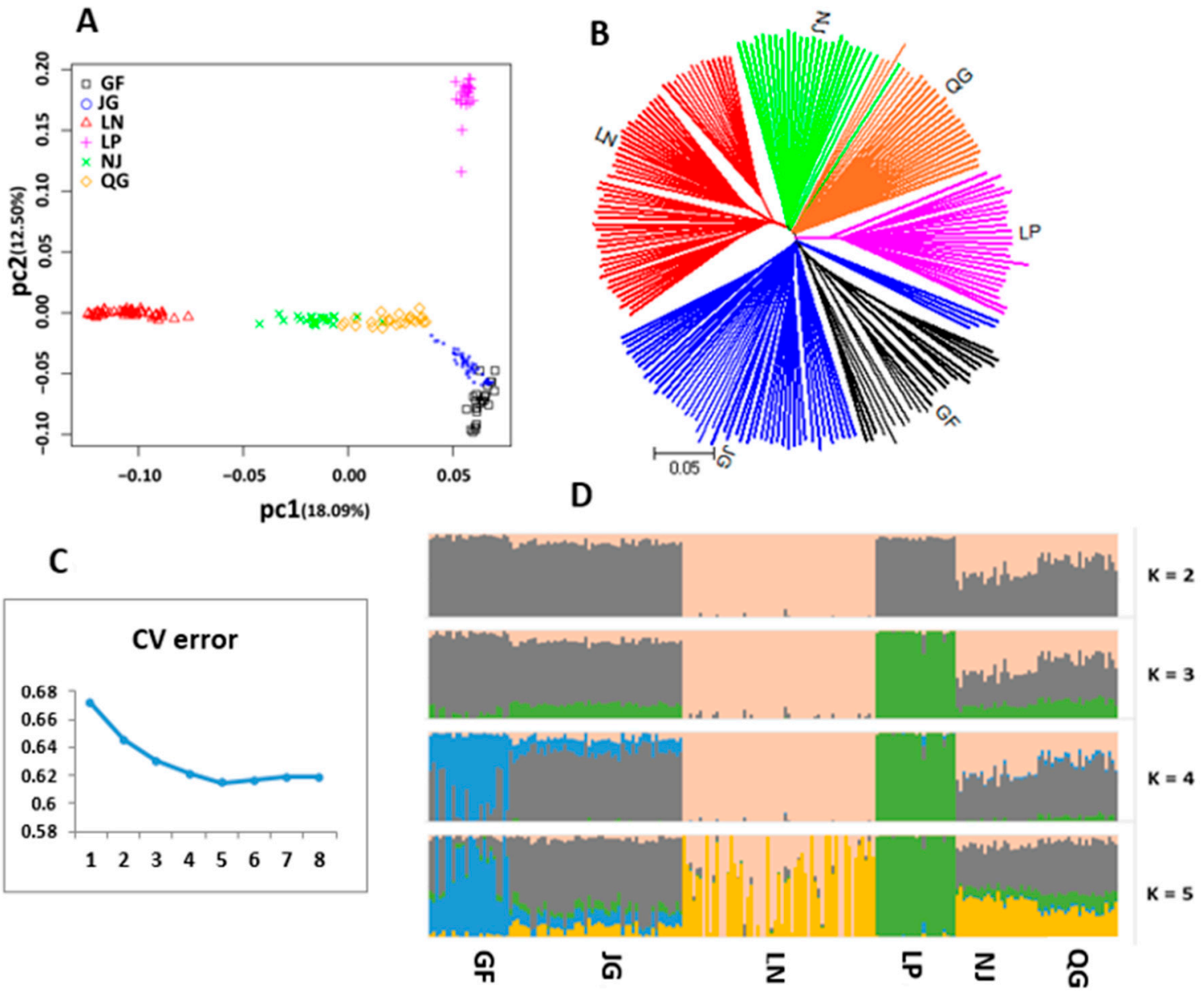

Figure 11. Phylogenetic relationships of six goat populations. (A) Principal component analysis of 204 individuals. (B) Neighbor-joining phylogenetic tree of goat breeds. (C) Cross-validation of six goat populations. (D) Genome-wide admixtures inferred by ADMIXTURE software. 


\subsubsection{Genome-Wide Selective Sweep Analysis in High-Fecundity Goat Breeds (High vs. Low)}

The regions falling within the upper $1 \%$ of the empirical distribution of $F_{\mathrm{ST}}\left(F_{\mathrm{ST}}>0.261025\right)$ and nucleotide diversity $(\pi)$ were scanned as candidate regions under selection. Based on the reference genome annotation, a gene that overlapped with an outlier of the top $1 \%$ of both the $F_{\mathrm{ST}}$ and $\pi$ estimates was deemed to be candidate gene under positive selection. We identified 460 candidate loci, of which a subset of 54 loci harbored 86 putative candidate genes with the signature of positive selection (Table S5). To improve confidence, we also scanned the top $0.1 \%$ of XP-EHH estimations. We found two candidate genes were highly differentiated between the high and low-reproduction groups according to three approaches used (Figure 12, Figure 13) that might have experienced strong positive selection. Based on their biological functions, associated pathways, and information from published studies, several genes were found possibly responsible for the reproduction trait in goats and are thus annotated in the Manhattan plot of $F_{\mathrm{ST}}, \log _{2}(\theta \pi$ ratio) and XP-EHH (Figure 13). Table 5 shows the potential candidate genes with their loci.

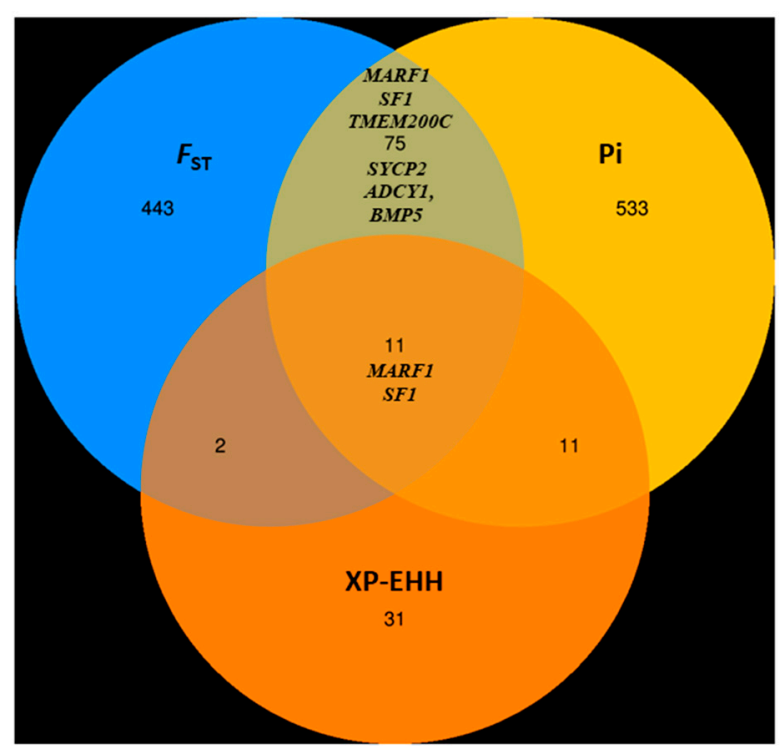

Figure 12. Venn diagram showing the overlapping genes in the top $1 \%$ of population differentiation index $\left(F_{\mathrm{ST}}\right)$, nucleotide diversity $(\pi)$ and $0.1 \%$ of Cross-population extended haplotype homozygosity $(\mathrm{XP}-\mathrm{EHH})$.

Table 5. Genomic loci containing the potential candidate genes.

\begin{tabular}{ccccccc}
\hline Chromosome & Position & $\boldsymbol{F}_{\mathbf{S T}}$ & $\mathbf{Z F}_{\mathbf{S T}}$ & $\boldsymbol{\pi}$ & XP-EHH & Gene Name \\
\hline 25 & $13,838,569$ & 0.419 & 6.129 & 5.002 & 3.486 & Meiosis arrest female 1 (MARF1) \\
29 & $43,352,393$ & 0.305 & 4.262 & 3.450 & 2.903 & Steroidogenic factor-1 (SF1) \\
24 & $39,657,370$ & 0.306 & 4.281 & 2.409 & & Transmembrane Protein 200C \\
13 & $55,939,729$ & 0.329 & 4.647 & 2.346 & & (TMEM200C) \\
4 & $43,874,883$ & 0.301 & 4.193 & 2.829 & & Synaptonemal Complex Protein 2 (SYCP2) \\
23 & $44,074,688$ & 0.286 & 3.946 & 2.635 & & Adenylate cyclase 1 (ADCY1) \\
& & & & & & Bone Morphogenetic Protein 5 (BMP5) \\
\hline
\end{tabular}



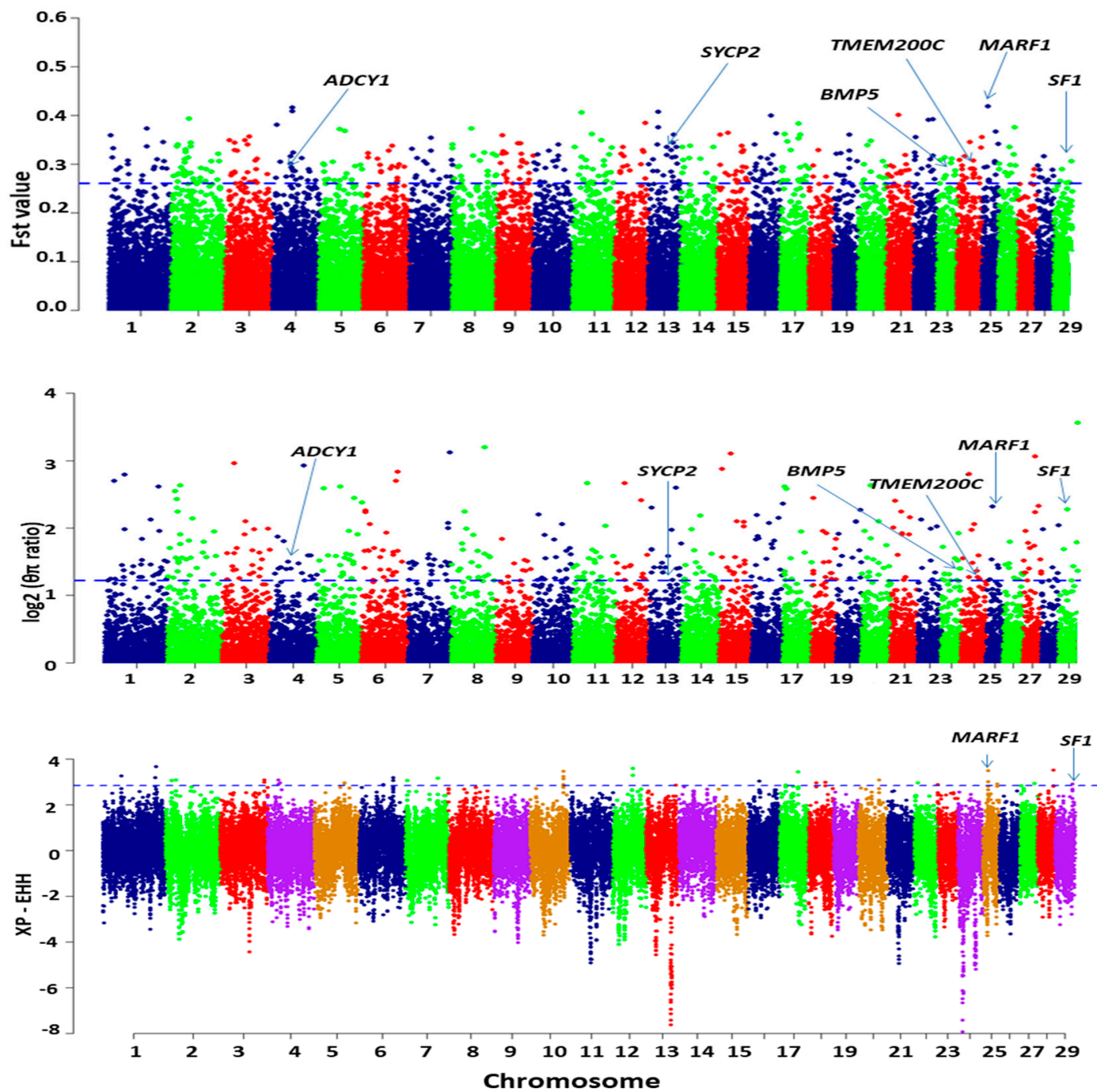

Figure 13. Selection signature detection in the high fecundity group of goat populations. Dotted lines indicate the top $1 \%$ values of $F_{\mathrm{ST}}$, and $\pi$, and $0.1 \%$ values of $\mathrm{XP}-\mathrm{EHH}$.

\subsubsection{GO Annotation and KEGG Pathway of the Target Genes}

Functional enrichment of the target genes revealed that the genes were significantly enriched in 20 GO terms in molecular functions, 103 terms in biological processes, and 49 terms in cellular components (Table S6). The most significant GO terms were found in metabolic processes, regulation of biological processes, developmental processes, and reproductive processes in BP, binding (GO: 0005488) in molecular functions, and intracellular organelles (GO: 0043229) in cellular components (Figure 14A).

A total of 25 KEGG pathways were found to be significantly enriched at the threshold $(p<0.05)$, although 93 pathways were involved with these genes. The most enriched KEGG pathways included "bile secretion", "cell cycle", "p53 signaling pathway", "Mitogen-activated protein kinase (MAPK) signaling pathway", "retrograde endocannabinoid signaling", "hippo signaling pathway", "oxytocin signaling pathway", "pathways in cancer" and "ovarian steroidogenesis" (Figure 14B, Table S7). 

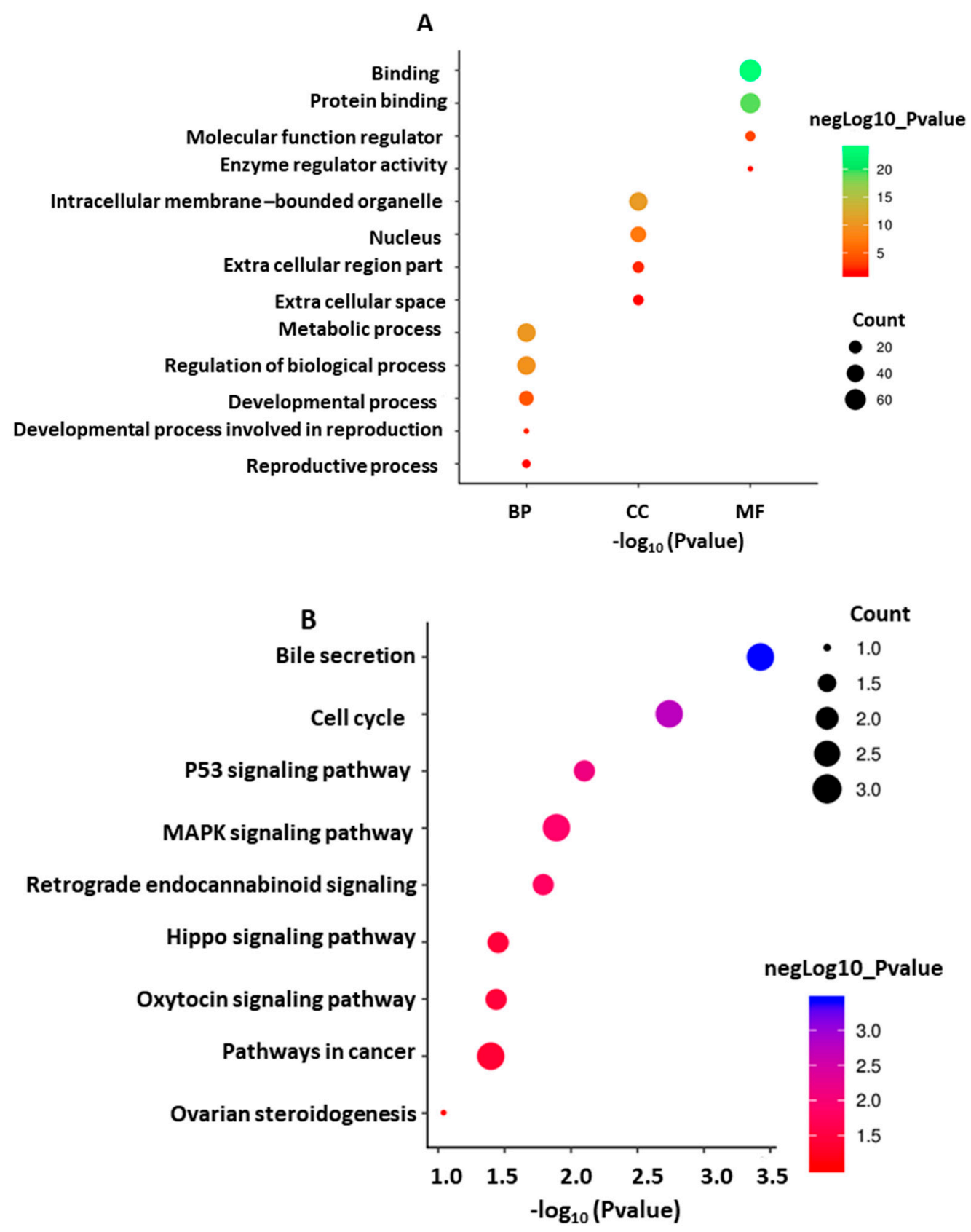

Figure 14. The bubble chart of (A) gene ontology (GO) terms and (B) Kyoto Encyclopedia of Genes and Genomes (KEGG) pathways of the selected genes.

\section{Discussion}

The detection of selection footprints in the genomic region has the potential to be used to identify genes and mutations associated with economically important phenotypic traits of livestock species. As the level of genetic diversity represents the raw materials for breed improvement, elucidating the genetic diversity of a population can provide insights for improving breeding strategies. $\mathrm{ROH}$, $\mathrm{HBD}$, and $\mathrm{N}_{e}$ are useful tools for exploring genetic diversity, providing information about population demographics evolution over time, and predicting underlying genome architecture. The developed genome-wide goat SNP arrays have become the marker of choice for investigating underlying genetic diversity, inferring population demography, and mapping genomic regions subject to selection [36]. In this study, we examined the genome-wide runs of homozygosity, effective population size, and signature of positive selection in six Chinese goat populations using goat SNP 50 K chips. 
Our findings showed that the ROHs are frequent across the populations. The distribution of ROHs per chromosome displayed a specific pattern: the greatest number was found in the first three chromosomes, the $\mathrm{ROH}$ number tended to decrease with decreasing chromosome length, and the smallest number was found on chromosome 28, consisting of 284 segments. Our results are consistent with those reported for Valle del Belice sheep [37]. The percentage of coverage per chromosome was quite different in the different populations, which suggests it may be breed-specific.

The higher sum of $\mathrm{ROH}$ length across the GF goats indicated their lower level of genetic diversity. In general, all breeds showed their majority of average ROHs in the $0-3 \mathrm{Mb}$ length class, which is in agreement with the results obtained for Spanish goats [34]. A different $\mathrm{ROH}$ distribution pattern was noted for GF goats, which displayed a high mean $\mathrm{ROH}$ in the long length category $(>30 \mathrm{Mb})$, which is indicative of demographic decline and recent inbreeding [38]. Thus, the accumulation of long ROHs in the genome of the GF breed enables them to carry deleterious mutations in homozygous form [39]. The highest value of $F_{\mathrm{ROH}}$ in GF, suggesting high inbreeding in this goat breed, is the signature of the extensive use of few bucks within herds. Consequently, widespread mating between relatives occurred, which might have contributed to the high proportion of fixed alleles, resulting in low genetic diversity in the GF population. Attention should be paid to this goat breed to prevent a loss of goat genetic resources. A large contribution to autozygosity was observed in GF goats in the HBD class, tracing back to different generations, which indicates a reduced effective population size, possibly due to a bottleneck or the founder effect. A similar trend in autozygosity was observed for sheep [40]. The limited amount of autozygosity in JG and QG goat populations suggested a large $N_{e}$ in the past. We found a declining trend in $N_{e}$ from 1000 to 100 generations ago across the studied populations. This decreasing trend in $N_{e}$ might be associated with human migration with goats that subsequently led to breed formation. The most rapid decline in $N_{e}$ was observed over the last 100 generations in all breeds, suggesting that significant bottlenecks occurred at the time of domestication, breed formation, and selection within these breeds [41,42]. The elevated $N_{e}$ estimates in JG, LP, NJ, and QG in all generations compared with GF and LN might be due to population admixture within these breeds. Brito and Jafarikia [43] estimated the $N_{e}$ in Australian and Canadian goats and found a similar trend of declining $N_{e}$ over the past generations, and an accelerated decline in $N_{e}$ over the past 100 generations. Comparatively, a lower $N_{e}$ was found in GF and LN, with estimated values were 42 and 85, respectively, 13 generations ago, which could be due to intensive selection pressure or artificial insemination used to develop these breeds. $N_{e}$ values for Argentinian, French, and South African goat breeds were reported to be 57,67 , and 93, respectively, at 10 generations ago [44], which is consistent with our findings. The $N_{e}$ slope for GF and LN in Figure 10b indicates constantly decreasing population sizes for these breeds, suggesting that action is needed to maintain a sufficiently large $N_{e}$.

All the pairwise $F_{\mathrm{ST}}$ values between populations were statistically significant $(p<0.05)$. The pair-wise $F_{\mathrm{ST}}$ values ranged from 0.02094 (NJ-QG) to 0.18172 (LN-LP), revealing the least differentiation between NJ and QG, whereas the highest differentiation was between LN and LP. In terms of genetic distance, $N$ and $Q G$ are most closely related $\left(D_{R}=0.02116\right)$, whereas $L N$ and LP appear most distinct $\left(D_{R}=0.20055\right)$, which is possibly due to shared common ancestry between NJ and QG and the geographical isolation of LP goats from other breeds. The LN is the most popular goat breed due to its cashmere trait and has experienced strong selection pressure. The LP breed is traditionally reared in the Yunnan province, in the southwest part of China. The Hengduan Mountains in between the Qinghai-Tibet Plateau and Yunnan-Guizhou Plateau have restricted livestock gene flow between Southwestern and Northern China. The results are similar to those reported for Ethiopian sheep breeds [45]. The AMOVA revealed that $89.61 \%$ of the genetic variation was within populations and $9.68 \%(p<0.001)$ was between populations. This is a little higher than the within-population variation and slightly lower than between-population variation $(87.86 \%$ and $11.86 \%)$ observed for South African, French, and Argentinian goat populations [44], and similar ( $89.83 \%$ and $7.49 \%$, respectively) to South African and Italian goat breeds in terms of within breed variation, as reported by Nicoloso and Bomba [13]. 


\subsection{Candidate Genes in the ROH Regions}

In this paper, we do not discuss all the genomic regions associated with a high $\mathrm{ROH}$ frequency; we focused on some selected regions that showed associations with the reproductive traits in goats. We identified eight genes reported to be associated with the reproductive traits of goats (Table 6) of which two genes (MARF1 and SYCP2) overlapped with the genes identified by the selection signature. The GDF9 gene was identified on chromosome 7 of GF goats, which plays a crucial role in early folliculogenesis in female mammals [46]. GDF7, which is the member of the BMP family and is required for seminal vesicle development, was detected on chromosome 11 [47]. INHA, located on chromosome 2, was reported as a candidate gene for litter size in goats [48]. We identified another gene, MTHFSD, on chromosome 18 of LP goats, which was reported to be involved in the variation of litter size [49]. MARF1, which is essential for the development of competent oocytes for successful fertilization, was detected on chromosome 25 [50]. On chromosome 13 of GF goats, we identified the SYCP2 gene, which is required for maintaining normal male and female fertility [51]. Another two genes (TMEM200C and ADCY1), which we identified by selection signature detection, were also close to the $\mathrm{ROH}$ region and involved in reproductive functions.

Table 6. List of candidate genes located in genomic regions with a high frequency of ROHs associated with goat reproductive traits.

\begin{tabular}{cccccrc}
\hline Breed & CHR & Start (bp) & End (bp) & Gene Symbol & $\begin{array}{c}\text { Distance between ROH } \\
\text { Region and Gene }\end{array}$ & Function \\
\hline GF & 7 & $63,446,436$ & $66,314,871$ & GDF9* & $2.57 \mathrm{Mb}$ & Reproduction \\
GF & 11 & $76,923,989$ & $77,967,512$ & $G D F 7^{*}$ & $0.98 \mathrm{Mb}$ & Reproduction \\
GF & 2 & $27,707,065$ & $29,287,557$ & $I N H A^{*}$ & $0.60 \mathrm{Mb}$ & Reproduction \\
LP & 18 & $13,400,847$ & $14,834,169$ & $M T H F S D^{*}$ & $0.35 \mathrm{Mb}$ & Reproduction \\
LP & 25 & $13,070,993$ & $14,141,921$ & $M A R F 1^{*}$ & $2.48 \mathrm{Mb}$ & Reproduction \\
GF & 13 & $53,460,986$ & $56,246,114$ & $S Y C P 2^{*}$ & Reproduction \\
JG & 24 & $40,941,530$ & $42,250,672$ & TMEM $200 C$ & Mb & Reproduction \\
LP & 4 & $41,890,323$ & $42,021,443$ & $A D C Y 1$ & $2.01 \mathrm{Mb}$ & Reproduction \\
\hline
\end{tabular}

** The distance between genes and $\mathrm{ROH}$ regions was calculated by subtracting the starting coordinate of the $\mathrm{ROH}$ region from the starting coordinate of the gene; ${ }^{*}$ Candidate genes are located in the $\mathrm{ROH}$ region.

\subsection{Signature of Selection}

For selection signature scanning, the six goat populations were divided into two groups based on their genetic pattern and fecundity rate collected from the records and literature. Phylogenic analyses supported the relatedness among the breeds of the high fecundity group and clearly separated the low from the high group. A strong genetic relationship was observed between NJ and QG as they are grouped closely in the neighbor-joining tree, indicating a high degree of gene flow due to being near to each other in geographic location. The tree also showed that the GF and JG breeds form a group in the same clade, suggesting that these goat breeds share common ancestry. This result is supported by the PCA, where JG and GF are clustered together. Population admixture analysis generated some signals of admixture and underlying genetic relationships among the populations. The high fecundity group included JG, GF, LP, and NJ ( 200\%), which have a distinct pattern, although the NJ goat was admixed with QG goat in the admixture result. The low fecundity group contained LN and QG ( $100 \%)$, and the LN goat was separated from the others in all the phylogenetic analyses.

Selective sweep based on population differentiation $\left(F_{\mathrm{ST}}\right)$ and nucleotide diversity $(\pi)$ for the high and low reproduction groups of goat breeds was detected. To minimize the false positive, we selected only the overlapping genes on the top $1 \%$ of $F_{\mathrm{ST}}$ and Pi approaches. To find the high-quality candidate genes, we also scanned $0.1 \%$ empirical distribution of XP-EHH estimates and selected the overlapping genes under these three approaches as strong candidate genes. Candidate genes associated with reproduction were identified in different genomic loci under selective sweep. Locus 13,838,569 of chromosome 25 had the highest $F_{\mathrm{ST}}$ value $\left(F_{\mathrm{ST}}=0.419, \mathrm{Z} F_{\mathrm{ST}}=6.129\right)$ and an elevated $\pi$ ratio and $\mathrm{XP}$-EHH $(\theta \pi$ ratio $=5.00, \mathrm{XP}-\mathrm{EHH}=3.486$; Table 5$)$. Based on the genome annotation, this locus 
encompasses MARF1 (Meiosis arrest female 1), which is required for the process of controlling meiosis and the development of healthy offspring. Accurate completion of the meiotic process, cytoplasmic maturational events, and genomic integrity is essential for the production of oocytes suitable for fertilization and embryogenesis. Genetic control of these events is vital for successful reproduction. A previous study showed that MARF1 is essential for the development of fertilization competent oocyte, and mutations lead to female infertility in mammals [50]. TMEM200C (Transmembrane Protein $200 C)$ was identified at locus $39,657,370$ in chromosome $24\left(F_{\mathrm{ST}}=0.306, Z F_{\mathrm{ST}}=4.281, \theta \pi\right.$ ratio $\left.=2.409\right)$. TMEM200C is commonly involved in the communication of a cell with its external environment. A Genome-Wide Association Studies reported that an SNP in the proximity of the TMEM200C gene is significantly associated with the twinning rate in Maremmana cattle [52]. The candidate gene SF1 (Steroidogenic factor- 1$)$, located at locus $43,352,393$ on chromosome $29\left(F_{\mathrm{ST}}=0.305, Z F_{\mathrm{ST}}=4.262\right.$, $\theta \pi$ ratio $=3.450, \mathrm{XP}-\mathrm{EHH}=2.903)$, is required for the formation of gonads [53] and to maintain normal reproduction [54]. The distant locus 55,939,729 of chromosome 13 showed high differentiation $\left(F_{\mathrm{ST}}=0.329, \mathrm{ZF} F_{\mathrm{ST}}=4.647, \theta \pi\right.$ ratio $\left.=2.346\right)$ and was detected as a putative selection signal. This locus contains SYCP2 (Synaptonemal Complex Protein 2), which is a proteinaceous structure required for the normal meiotic fusion of oocytes and spermatocyte formation and to maintain normal male and female fertility. A study suggested that $S Y C P 2 L$, which is the paralog of $S Y C P 2$, plays a significant role in the survival of oocytes and regulates reproductive aging in females [51]. We detected $A D C Y 1$ (Adenylate cyclase 1), which showed evidence for positive selection for reproduction and is located at locus $43,874,883$ on chromosome $4\left(F_{\mathrm{ST}}=0.301, \mathrm{Z} F_{\mathrm{ST}}=4.193, \theta \pi\right.$ ratio $\left.=2.829\right)$. The candidate gene $A D C Y 1$ encodes adenylate cyclase 1, which is involved in the formation of cyclic adenosine monophosphate (cAMP) cyclizing AMP, which subsequently partakes in oocyte meiotic arrest and suppresses its resumption [55]. BMP5 (Bone Morphogenetic Protein 5) was identified in the proximity of locus 44,074,688 $\left(F_{\mathrm{ST}}=0.286, Z F_{\mathrm{ST}}=3.946, \theta \pi\right.$ ratio $\left.=2.635\right)$ of chromosome 23 and is involved in the hippo signaling pathway, which plays a significant role in controlling follicular growth and ovulation in livestock [56]. BMP5 belongs to the BMP subfamily and plays a crucial role in ovarian folliculogenesis by enhancing proliferation of granulosa cells, which is associated with the growing female gamete in the mammalian ovary [57], indicating that this gene is more likely to be under selection. MARF1 and SF1 polymorphisms are noticeable as they are highly differentiated between the high- and low-reproduction groups under our three approaches used.

Functional enrichment analysis is used to explore a controlled vocabulary to extensively describe the characteristics of genes and gene products. According to our enrichment analysis results, several GO and pathways were directly or indirectly involved with the reproduction of animals, although some of the pathways could not pass the significance threshold $(p<0.05)$. The selected genes were mostly enriched in biological processes, including metabolic processes, regulation of biological processes, developmental processes, and reproductive processes. The most significant GO terms were found binding (GO: 0005488) in molecular functions and intracellular organelles (GO: 0043229) in cellular components. The pathway analysis revealed the involvement of selected genes in reproduction. The mitogen-activated protein kinase (MAPK) pathway has important roles in oocyte maturation and eventually in ovulation by regulating cyclic guanosine monophosphate production and its transportation to the oocyte [58,59]. Other enriched pathways, such as bile secretion (hsa04976), retrograde endocannabinoid signaling (hsa04723), the hippo signaling pathway (hsa04390), the oxytocin signaling pathway (hsa04921), and ovarian steroidogenesis (hsa04913), play significant roles in the processes of oocyte maturation, regulation of follicular growth, and ovulation in livestock [56]. Although some of the candidate genes were enriched in none of the significant pathways, these genes were deemed to be candidate genes for reproduction due to their biological functions and information from published studies. 


\section{Conclusions}

In this study, we investigated the patterns of homozygosity and the signature of positive selection in six Chinese goat populations. Demographic decline, the highest level of inbreeding and severe founder effects are the signatures of an increased level of homozygosity in Guangfeng goat while population admixture is associated with the opposite effect in other goat breeds. The existence of two genes in the $\mathrm{ROH}$ regions coincided with the regions detected by the selection signal demonstrated that the homozygosity is not solely the result of demography instead of positive selection. Several genes were identified as candidate genes by $F_{\mathrm{ST}}, \pi$ and XP-EHH algorithm of which MARF1 and SF1 genes are highly differentiated between high and low reproduction groups according to the three approaches used. The information about the genetic status and the identified genes under positive selection may be useful for further genetic improvement and setting conservation program for these important goat breeds. Further studies relying on high-density markers are necessary to evaluate the level of genetic autozygosity and signature of selection of the goat breed.

Supplementary Materials: The following supplementary materials are available online at http://www.mdpi.com/ 2073-4425/10/11/938/s1. Table S1: A short description (Breed name, sample size, fecundity rate, demography and breeding practices, average altitude and agro-ecology) of six goat populations. Table S2: Average sum of ROH content; Table S3: Genomic Regions with a High ROH Frequency; Table S4. Analysis of molecular variance (AMOVA) among six Chinese goat populations; Table S5: Overlapping genes of top $1 \% F_{\mathrm{ST}}, \pi$ ratio and $0.1 \%$ XP-EHH; Table S6: GO Term; Table S7: KEGG Pathway.

Author Contributions: Conceptualization, R.I.; investigation, R.I.; methodology, R.I., H.B., A.A.; software, R.I.; data curation, R.I., X.L.; writing—original draft preparation, R.I.; writing—review and editing, R.I., Y.L., X.L., G.G.; visualization, R.I., X.L. and Y.L.; supervision, Q.M. and Y.M.; project administration, R.I. and Y.M.; funding acquisition, Y.M.

Funding: This research was funded by the National Natural Science Foundation of China (No.31601910), the earmarked fund for Modern Agro-industry Technology Research System (CARS-39-01), Ningxia Special Project of Agricultural Breeding (The key technology integration and demonstration of high-quality meat production of Tan sheep, 2018BBF02016) and the Basic R\&D Fund for the Central Level Scientific Research Institute (Y2017JC03, Y2018PT68).

Acknowledgments: We are grateful to the editors and the two anonymous reviewers for their insightful comments and constructive suggestions that helped us greatly to improve our manuscript.

Conflicts of Interest: The authors declare that there are no competing interests with respect to the authorship or publication of this article.

\section{References}

1. Liu, G.; Zhao, Q.; Lu, J.; Sun, F.; Han, X.H.; Zhao, J.; Feng, H.; Wang, K.; Liu, C. Insights into the genetic diversity of indigenous goats and their conservation priorities. Asian Australas. J. Anim. Sci. 2019, 32, 1501-1510. [CrossRef] [PubMed]

2. Brito, L.F.; Kijas, J.W.; Ventura, R.V.; Sargolzaei, M.; Porto-Neto, L.R.; Cánovas, A.; Feng, Z.; Jafarikia, M.; Schenkel, F.S. Genetic diversity and signatures of selection in various goat breeds revealed by genome-wide SNP markers. BMC Genom. 2017, 18, 229. [CrossRef] [PubMed]

3. de Cara, M.Á.R.; Villanueva, B.; Toro, M.Á.; Fernández, J. Using genomic tools to maintain diversity and fitness in conservation programmes. Mol. Ecol. 2013, 22, 6091-6099. [CrossRef] [PubMed]

4. Bosse, M.; Megens, H.-J.; Madsen, O.; Crooijmans, R.P.; Ryder, O.A.; Austerlitz, F.; Groenen, M.A.; De Cara, M. Ángeles R. Using genome-wide measures of coancestry to maintain diversity and fitness in endangered and domestic pig populations. Genome Res. 2015, 25, 970-981. [CrossRef]

5. Ferenčaković, M.; Hamzić, E.; Gredler, B.; Solberg, T.R.; Klemetsdal, G.; Curik, I.; Sölkner, J. Estimates of autozygosity derived from runs of homozygosity: Empirical evidence from selected cattle populations. J. Anim. Breed. Genet. 2013, 130, 286-293. [CrossRef]

6. Peripolli, E.; Munari, D.P.; Silva MV, G.B.; Lima AL, F.; Irgang, R.; Baldi, F. Runs of homozygosity: Current knowledge and applications in livestock. Anim. Genet. 2017, 48, 255-271. [CrossRef]

7. Pemberton, T.J.; Absher, D.; Feldman, M.W.; Myers, R.M.; Rosenberg, N.A.; Li, J.Z. Genomic Patterns of Homozygosity in Worldwide Human Populations. Am. J. Hum. Genet. 2012, 91, 275-292. [CrossRef] 
8. Szmatoła, T.; Gurgul, A.; Ropka-Molik, K.; Jasielczuk, I.; Ząbek, T.; Bugno-Poniewierska, M. Characteristics of runs of homozygosity in selected cattle breeds maintained in Poland. Livest. Sci. 2016, 188, 72-80. [CrossRef]

9. Bertrand, A.R.; Kadri, N.K.; Flori, L.; Gautier, M.; Druet, T. RZooRoH: An R package to characterize individual genomic autozygosity and identify homozygous-by-descent segments. Methods Ecol. Evol. 2019, 10, 860-866. [CrossRef]

10. Wright, S. Size of population and breeding structure in relation to evolution. Science 1938, 87, 430-431.

11. Liu, S.; He, S.; Chen, L.; Li, W.; Di, J.; Liu, M. Estimates of linkage disequilibrium and effective population sizes in Chinese Merino (Xinjiang type) sheep by genome-wide SNPs. Genes Genom. 2017, 39, 733-745. [CrossRef] [PubMed]

12. Shin, D.-H.; Cho, K.-H.; Park, K.-D.; Lee, H.-J.; Kim, H. Accurate Estimation of Effective Population Size in the Korean Dairy Cattle Based on Linkage Disequilibrium Corrected by Genomic Relationship Matrix. Asian-Australasian J. Anim. Sci. 2013, 26, 1672-1679. [CrossRef] [PubMed]

13. Nicoloso, L.; Bomba, L.; Colli, L.; Negrini, R.; Milanesi, M.; Mazza, R.; Sechi, T.; Frattini, S.; Talenti, A.; Coizet, B.; et al. Genetic diversity of Italian goat breeds assessed with a medium-density SNP chip. Genet. Sel. Evol. 2015, 47, 62. [CrossRef] [PubMed]

14. Tao, F.; Li, Z.Z.-X. DNA polymorphism of introns 1 and 2 of Prolactin Receptor Gene and its association with litter size in goats. Anim. Sci. Pap. Rep. 2011, 29, 343-350.

15. Guan, D.; Luo, N.; Tan, X.; Zhao, Z.; Huang, Y.; Na, R.; Zhang, J.; Zhao, Y. Scanning of selection signature provides a glimpse into important economic traits in goats (Capra hircus). Sci. Rep. 2016, 6, 36372. [CrossRef] [PubMed]

16. Wei, C.; Lu, J.; Xu, L.; Liu, G.; Wang, Z.; Zhao, F.; Zhang, L.; Han, X.; Du, L.; Liu, C. Genetic Structure of Chinese Indigenous Goats and the Special Geographical Structure in the Southwest China as a Geographic Barrier Driving the Fragmentation of a Large Population. PLoS ONE 2014, 9, e94435. [CrossRef] [PubMed]

17. Du, L. Animal Genetic Resources in China: Sheep and Goats; China Agricultural Publishing House: Beijing, China, 2011; pp. 235-425.

18. Liu, Q.; Chen, P.; He, K.; Kilpatrick, C.W.; Liu, S.-Y.; Yu, F.-H.; Jiang, X.-L. Phylogeographic Study of Apodemus ilex (Rodentia: Muridae) in Southwest China. PLoS ONE 2012, 7, e31453. [CrossRef]

19. Yuan, J.; Cheng, F.; Zhou, S. Genetic Structure of the Tree Peony (Paeonia rockii) and the Qinling Mountains as a Geographic Barrier Driving the Fragmentation of a Large Population. PLoS ONE 2012, 7, e34955. [CrossRef]

20. Purcell, S.; Neale, B.; Todd-Brown, K.; Thomas, L.; Ferreira, M.A.R.; Bender, D.; Maller, J.; Sklar, P.; De Bakker, P.I.W.; Daly, M.J.; et al. PLINK: A Tool Set for Whole-Genome Association and Population-Based Linkage Analyses. Am. J. Hum. Genet. 2007, 81, 559-575. [CrossRef]

21. Chang, C.C.; Chow, C.C.; Tellier, L.C.; Vattikuti, S.; Purcell, S.M.; Lee, J.J. Second-generation PLINK: Rising to the challenge of larger and richer datasets. GigaScience 2015, 4, 7. [CrossRef]

22. Kumar, C.; Song, S.; Dewani, P.; Kumar, M.; Parkash, O.; Ma, Y.; Malhi, K.K.; Yang, N.; Mwacharo, J.M.; He, X.; et al. Population structure, genetic diversity and selection signatures within seven indigenous Pakistani goat populations. Anim. Genet. 2018, 49, 592-604. [CrossRef] [PubMed]

23. Al-Mamun, H.A.; Clark, S.A.; Kwan, P.; Gondro, C. Genome-wide linkage disequilibrium and genetic diversity in five populations of Australian domestic sheep. Genet. Sel. Evol. 2015, 47, 90. [CrossRef]

24. McQuillan, R.; Leutenegger, A.-L.; Abdel-Rahman, R.; Franklin, C.S.; Pericic, M.; Barac-Lauc, L.; Smolej-Narancic, N.; Janicijevic, B.; Polašek, O.; Tenesa, A.; et al. Runs of Homozygosity in European Populations. Am. J. Hum. Genet. 2008, 83, 359-372. [CrossRef] [PubMed]

25. NCBI. Gene Information for Ars1 Gcf Gz. 2019. Available online: https://www.ncbi.nlm.nih.gov/genome/ ?term=goat/GCF_001704415.1_ARS1_genomic_gff.gz (accessed on 10 October 2019).

26. Solé, M.; Gori, A.-S.; Faux, P.; Bertrand, A.; Farnir, F.; Gautier, M.; Druet, T. Age-based partitioning of individual genomic inbreeding levels in Belgian Blue cattle. Genet. Sel. Evol. 2017, 49, 92. [CrossRef] [PubMed]

27. Barbato, M.; Orozco-Terwengel, P.; Tapio, M.; Bruford, M.W. SNeP: A tool to estimate trends in recent effective population size trajectories using genome-wide SNP data. Front. Genet. 2015, 6, 109. [CrossRef]

28. Sved, J. Linkage disequilibrium and homozygosity of chromosome segments in finite populations. Theor. Popul. Biol. 1971, 2, 125-141. [CrossRef]

29. Excoffier, L.; Lischer, H.E.L. Arlequin suite ver 3.5: A new series of programs to perform population genetics analyses under Linux and Windows. Mol. Ecol. Resour. 2010, 10, 564-567. [CrossRef] 
30. Zheng, X.; Levine, D.; Shen, J.; Gogarten, S.M.; Laurie, C.; Weir, B.S. A high-performance computing toolset for relatedness and principal component analysis of SNP data. Bioinformatics 2012, 28, 3326-3328. [CrossRef]

31. Tamura, K.; Peterson, D.; Peterson, N.; Stecher, G.; Nei, M.; Kumar, S. MEGA5: Molecular Evolutionary Genetics Analysis Using Maximum Likelihood, Evolutionary Distance, and Maximum Parsimony Methods. Mol. Biol. Evol. 2011, 28, 2731-2739. [CrossRef]

32. Alexander, D.H.; Novembre, J.; Lange, K. Fast model-based estimation of ancestry in unrelated individuals. Genome Res. 2009, 19, 1655-1664. [CrossRef]

33. Weir, B.S.; Cockerham, C.C. Estimating F-Statistics for the Analysis of Population Structure. Evolution 1984, 38, 1358-1370. [PubMed]

34. Guo, J.; Tao, H.; Li, P.; Li, L.; Zhong, T.; Wang, L.; Ma, J.; Chen, X.; Song, T.; Zhang, H. Whole-genome sequencing reveals selection signatures associated with important traits in six goat breeds. Sci. Rep. 2018, 8, 10405. [CrossRef] [PubMed]

35. Purfield, D.C.; Berry, D.P.; McParland, S.; Bradley, D.G. Runs of homozygosity and population history in cattle. BMC Genet. 2012, 13, 70. [CrossRef] [PubMed]

36. Kijas, J.W.; Townley, D.; Dalrymple, B.P.; Heaton, M.P.; Maddox, J.F.; McGrath, A.; Wilson, P.; Ingersoll, R.G.; McCulloch, R.; McWilliam, S.; et al. A Genome Wide Survey of SNP Variation Reveals the Genetic Structure of Sheep Breeds. PLoS ONE 2009, 4, e4668. [CrossRef] [PubMed]

37. Mastrangelo, S.; Tolone, M.; Sardina, M.T.; Sottile, G.; Sutera, A.M.; Di Gerlando, R.; Portolano, B. Genome-wide scan for runs of homozygosity identifies potential candidate genes associated with local adaptation in Valle del Belice sheep. Genet. Sel. Evol. 2017, 49, 84. [CrossRef] [PubMed]

38. Manunza, A.; Noce, A.; Serradilla, J.M.; Goyache, F.; Martínez, A.; Capote, J.; Delgado, J.V.; Jordana, J.; Muñoz, E.; Molina, A.; et al. A genome-wide perspective about the diversity and demographic history of seven Spanish goat breeds. Genet. Sel. Evol. 2016, 48, 52. [CrossRef]

39. Szpiech, Z.A.; Xu, J.; Pemberton, T.J.; Peng, W.; Zöllner, S.; Rosenberg, N.A.; Li, J.Z. Long Runs of Homozygosity Are Enriched for Deleterious Variation. Am. J. Hum. Genet. 2013, 93, 90-102. [CrossRef]

40. Druet, T.; Gautier, M. A model-based approach to characterize individual inbreeding at both global and local genomic scales. Mol. Ecol. 2017, 26, 5820-5841. [CrossRef]

41. Makina, S.O.; Taylor, J.F.; Van Marle-Koster, E.; Muchadeyi, F.C.; Makgahlela, M.L.; MacNeil, M.D.; Maiwashe, A. Extent of Linkage Disequilibrium and Effective Population Size in Four South African Sanga Cattle Breeds. Front. Genet. 2015, 6, 24. [CrossRef]

42. Daetwyler, H.D.; Pong-Wong, R.; Villanueva, B.; Woolliams, J.A. The Impact of Genetic Architecture on Genome-Wide Evaluation Methods. Genetics 2010, 185, 1021-1031. [CrossRef]

43. Brito, L.F.; Jafarikia, M.; Grossi, D.A.; Kijas, J.W.; Porto-Neto, L.R.; Ventura, R.V.; Salgorzaei, M.; Schenkel, F.S. Characterization of linkage disequilibrium, consistency of gametic phase and admixture in Australian and Canadian goats. BMC Genet. 2015, 16, 67. [CrossRef] [PubMed]

44. Visser, C.; Lashmar, S.F.; Van Marle-Köster, E.; Poli, M.A.; Allain, D. Genetic Diversity and Population Structure in South African, French and Argentinian Angora Goats from Genome-Wide SNP Data. PLoS ONE 2016, 11, e0154353. [CrossRef] [PubMed]

45. Edea, Z.; Dessie, T.; Dadi, H.; Do, K.-T.; Kim, K.-S. Genetic Diversity and Population Structure of Ethiopian Sheep Populations Revealed by High-Density SNP Markers. Front. Genet. 2017, 8, 218. [CrossRef] [PubMed]

46. Wang, X.; Yang, Q.; Wang, K.; Yan, H.; Pan, C.; Chen, H.; Liu, J.; Zhu, H.; Qu, L.; Lan, X. Two strongly linked single nucleotide polymorphisms (Q320P and V397I) in GDF9 gene are associated with litter size in cashmere goats. Theriogenology 2019, 125, 115-121. [CrossRef]

47. Settle, S.; Marker, P.; Gurley, K.; Sinha, A.; Thacker, A.; Wang, Y.; Higgins, K.; Cunha, G.; Kingsley, D.M. The BMP Family Member Gdf7 Is Required for Seminal Vesicle Growth, Branching Morphogenesis, and Cytodifferentiation. Dev. Biol. 2001, 234, 138-150. [CrossRef]

48. Hou, J.; An, X.; Li, G.; Wang, Y.; Song, Y.; Cao, B. Exploring polymorphisms and their effects on reproductive traits of the INHA and INH $\beta$ A genes in three goat breeds. Anim. Sci. J. 2012, 83, 273-278. [CrossRef] [PubMed]

49. Ran, X.-Q.; Pan, H.; Huang, S.-H.; Liu, C.; Niu, X.; Li, S.; Wang, J.-F. Copy number variations of MTHFSD gene across pig breeds and its association with litter size traits in Chinese indigenous Xiang pig. J. Anim. Physiol. Anim. Nutr. 2018, 102, 1320-1327. [CrossRef] 
50. Su, Y.-Q.; Sugiura, K.; Sun, F.; Pendola, J.K.; Cox, G.A.; Handel, M.A.; Schimenti, J.C.; Eppig, J.J. MARF1 regulates essential oogenic processes in mice. Science 2012, 335, 1496-1499. [CrossRef]

51. Zhou, J.; Stein, P.; Leu, N.A.; Chmátal, L.; Xue, J.; Ma, J.; Huang, X.; Lampson, M.A.; Schultz, R.M.; Wang, P.J. Accelerated reproductive aging in females lacking a novel centromere protein SYCP2L. Hum. Mol. Genet. 2015, 24, 6505-6514. [CrossRef]

52. Moioli, B.; Steri, R.; Marchitelli, C.; Catillo, G.; Buttazzoni, L. Genetic parameters and genome-wide associations of twinning rate in a local breed, the Maremmana cattle. Animal 2017, 11, 1660-1666. [CrossRef]

53. Barrionuevo, F.J.; Burgos, M.; Scherer, G.; Jiménez, R. Genes promoting and disturbing testis development. Histol. Histopathol. 2012, 27, 1361-1383. [PubMed]

54. Xu, Y.; Faulkner, L.D.; Hill, J.W. Cross-Talk between Metabolism and Reproduction: The Role of POMC and SF1 Neurons. Front. Endocrinol. 2012, 2, 98. [CrossRef] [PubMed]

55. Sun, M.-H.; Zheng, J.; Xie, F.-Y.; Shen, W.; Yin, S.; Ma, J.-Y. Cumulus Cells Block Oocyte Meiotic Resumption via Gap Junctions in Cumulus Oocyte Complexes Subjected to DNA Double-Strand Breaks. PLoS ONE 2015, 10, e0143223. [CrossRef] [PubMed]

56. Xu, S.-S.; Gao, L.; Xie, X.-L.; Ren, Y.-L.; Shen, Z.-Q.; Wang, F.; Shen, M.; EyPórsdóttir, E.; Hallsson, J.H.; Kiseleva, T.; et al. Genome-Wide Association Analyses Highlight the Potential for Different Genetic Mechanisms for Litter Size Among Sheep Breeds. Front. Genet. 2018, 9, 118. [CrossRef] [PubMed]

57. Pierre, A.; Pisselet, C.; Dupont, J.; Bontoux, M.; Monget, P. Bone Morphogenetic Protein 5 Expression in the Rat Ovary: Biological Effects on Granulosa Cell Proliferation and Steroidogenesis1. Biol. Reprod. 2005, 73, 1102-1108. [CrossRef] [PubMed]

58. Fan, H.Y.; Liu, Z.; Mullany, L.K.; Richards, J.S. Consequences of RAS and MAPK activation in the ovary: The good, the bad and the ugly. Mol. Cell. Endocrinol. 2012, 356, 74-79. [CrossRef] [PubMed]

59. Shkolnik, K.; Tadmor, A.; Ben-Dor, S.; Nevo, N.; Galiani, D.; Dekel, N. Reactive oxygen species are indispensable in ovulation. Proc. Natl. Acad. Sci. USA 2011, 108, 1462-1467. [CrossRef]

(C) 2019 by the authors. Licensee MDPI, Basel, Switzerland. This article is an open access article distributed under the terms and conditions of the Creative Commons Attribution (CC BY) license (http://creativecommons.org/licenses/by/4.0/). 